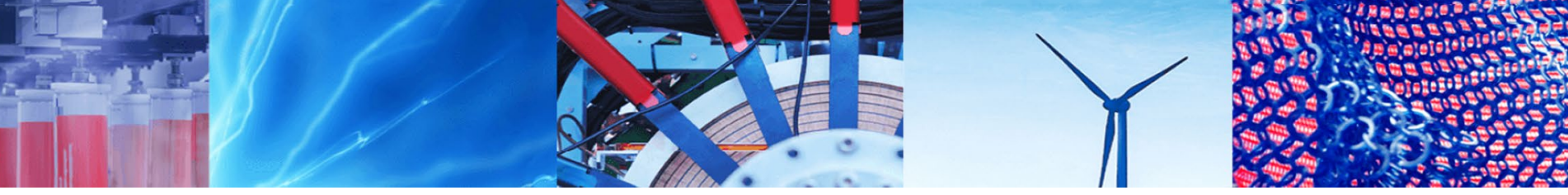

Review Paper

\title{
A review on graphene strain sensors based on fiber assemblies
}

\author{
Ting Wang ${ }^{1} \cdot$ Zhaofeng Ouyang $^{2} \cdot$ Fei Wang $^{1} \cdot$ Yixin Liu $^{1}$ (I)
}

Received: 28 October 2019 / Accepted: 30 March 2020 / Published online: 9 April 2020

(c) Springer Nature Switzerland AG 2020

\begin{abstract}
With the development of wearable electronic devices, electronic-textiles have attracted more and more attentions due to its widely applications in human movement monitoring, health and physical indicators monitoring (e.g. heartbeat, pulse, body temperature, limb movements, and vocalization). As a vital part of electronic textiles, graphene strain sensors that based on fiber assemblies (FGS) is of concern to the researchers due to its well integration of graphene with fibers assemblies, which will inevitably promote the rapid development and widespread applications of next-generation wearable electronic devices. Fiber assemblies exhibit outstanding ability of structural retention and fatigue resistance during wearing and washing, which can provide a large-area affiliated carrier and widespread service platform for the design and application of electronic-textiles. And the graphene endows electronic-textiles with good electrical and mechanical properties. Herein, the previous researches on fabrication, sensing mechanism, sensing performance indexes, and application field of FGS are systematically summarized. Furthermore, the potential difficulties and future development prospect of FGS are summarized and discussed. It is expected that this review is not only a summary of what has been achieved, but also provide a guideline for future development.
\end{abstract}

Keywords E-textiles · Fiber-assemblies · Graphene · Strain sensor

\section{Introduction}

With the appearance of "Google Glasses", 2012 was called "the first year of intelligent wearable devices." Intelligent wearable devices had been widely recognized and focused on smart terminal industry as the space for innovation in smart phones was gradually compressed and the market increment was approaching saturation. The intelligent wearable devices such as smart watches, smart glasses and smart wristbands have been widely used now for monitoring human movement, physical parameters, health indicators. The intelligent wearable devices are generally composed of a set of various functional modules (e.g. display devices, microprocessors, sensors, batteries, and communication units). Among all of these, the sensors play an important role, because the sensors could respond and transmit the change of human motion parameters, body and health indexes through the form of electrical signals. For example, the resistance of the sensing material could be changed by strain during human movement [1], the change of capacitance of the sensing material could be caused by human' pulse or heartbeat [2]. There still remain two problems, however, with sensors in current intelligent wearable devices. Firstly, the characteristic of rigidity of sensing materials limit the sufficient and effective interaction with curved surface of human body and leads to the distortion or inaccuracy of the collected electrical signals. Secondly, the sensing material does not have sufficient stretchability to meet the large strain requirements of the human body (strain range from 33 to $55 \%$ ) [3]. Replacement of rigid materials with flexible sensing materials is an effective way to solve the above problems $[4,5]$.

$\triangle$ Yixin Liu, liuyixin71@188.com | ${ }^{1}$ College of Textiles and Clothing, Qingdao University, Qingdao 266071, China. ${ }^{2}$ The Key Laboratory of Advanced Textile Materials and Manufacturing Technology of Ministry of Education, College of Materials and Textiles, Zhejiang Sci-Tech University, 928 Second Avenue, Xiasha Higher Education Park, Hangzhou 310018, China. 
Clothing, as one of the fiber assemblies, has been used for thousands of years due to its properties of flexibility, lightweight, stretchability, washability, and adaptability to the strain requirement while wearing. Meanwhile, it can fully interact with the curved surface of human body enduringly and has certain comfortable performance. Although these advantages of fiber assemblies can be used as a structural platform for wearable electronic device, what should be emphasized is to develop sustainable flexible systems called "electronic-textiles" [3], which provide high carrier mobility (electrical conductivity), mechanical stability and environmental stability. In the past few decades, a large number of attempts had been made to fabricate sensors suitable for "electronic-textiles" and two different methods of sensors had been developed. The one is that the sensor are fabricated by using conductive fibers which are made of conductive polymer, such as metal, carbon, piezoelectric materials, or conventional fibers whose surface covered by conductive materials, then processed into fiber assemblies structure $[6,7]$. The other is that, the sensors are fabricated by using the ordinary fiber assemblies structure as a motherboard and i MParting electronic functions on the surface of fabrics by dip-coating, printing or filtration $[8,9]$. No matter which of the above fabrication is used, first of all, the electrical conductivity of materials should be integrated with the fiber assemblies' structures (e.g. yarns [10], woven fabric [11], knitting fabric [12], nonwoven fabric [13].) These electrical conductive materials including organic polymers such as polyaniline (PANI) [14], polypyrrole (PPy) [15], poly3, 4-ethylene dioxythiophene (PEDOT) [16], as well as some inorganic carbon-based materials such as carbon black (CB) [17], carbon nanotubes (CNT) [18], graphene (GR) [19].

In recent years, graphene has attracted wide attention due to its tra-high electronic mobility $(15,000-200,000$ $\mathrm{cm}^{2} \mathrm{~V}^{-1} \mathrm{~s}^{-1}$ ) [20] and its excellent mechanical properties (Young's modulus of $1100 \mathrm{GPa}$ and tensile strength of $130 \mathrm{GPa}$ ) [21]. To combine graphene with other fiber forming polymers to make fibers and then process into fiber assemblies and to combine the graphene with off-theshelf fibers assemblies motherboard are the two mainly fabrications of graphene composite strain sensor based on fiber assemblies (FGS) for "electronic textiles". Ming Chao et al. reported a FGS based on skin-core structured fiber by using ultrafine graphenes as the skin and silk fibers as the core by dry-meyer-rod-coating process, the FGS with sensitivity of Gauge Factor (GF) 4.5 at strain $15 \%$ can be used to detect the human joint motions [22]. Xiaoting et al. using polyurethane yarns as substrate by coating graphene/poly vinyl alcohol as the conductive sheath, the prepared FGS with the sensitivity of GF 28.6 at 50\% strain is potential to monitor the human movement [10]. Liu et al. using woven Polydimethylsiloxane (PDMS) fabric as a substrate and growing graphene on the surface of fabric by chemical vapor deposition (CVD). the prepared FGS with high sensitivity of GF value 151 at $4.5 \%$ strain can be used for wireless musical instruments [23]. These studies also confirm that FGS has broad application prospects as electronic textiles.

Although FGS has been studied for many years and some achievements have been made, some questions still need to be further explored, such as how to improve the durability of FGS by improving the interfacial properties of graphene and fiber-forming polymers, how to improve the linearity of sensing sensitivity by designing a suitable fiber assemblies structure, how to reduce the response time and recovery time of sensors by mastering the relationship between viscoelastic characteristics of fiber polymer and sensing hysteresis. Herein, the previous studies on FGS have been reviewed systematically. The fabrication process of graphene and FGS, sensing mechanisms, performance and applications of the FGS are reviewed and discussed. At the same time, the existing challenges and the prospects for its future development are summarized.

\section{Graphene preparation}

Graphene is a single layer of carbon atoms densely arranged in a honeycomb lattice. The carbon-carbon bonds $\left(\mathrm{sp}^{2}\right.$ hybrid orbital) in graphene are about $0.142 \mathrm{~nm}$ in length. It's made up of benzene rings that have lost their hydrogen atoms [24]. Graphene have four carbon allotropes: graphite, carbon nanotubes, and fullerenes [25]. Graphene has been widely used as nanofillers for strain sensors due to its good mechanical and electrical property (stress of $130 \mathrm{GPa}$, conductivity of $7200 \mathrm{~S} \mathrm{~m}$ ) [26-30]. At present, there are three main methods for graphene preparation: Chemical vapor deposition (CVD) [31], Redox Process [32] and Liquid phase exfoliation (LPE) [33-35]. Among them, the redox method can also be regarded as a special way of LPE.

\subsection{CVD method}

CVD method is able to grow large-area, high-quality graphene films on metal surfaces by gaseous carbon sources. Metals, such as $\mathrm{Ni}, \mathrm{Fe}, \mathrm{Co}, \mathrm{Pt}$, and $\mathrm{Cu}$, are both catalysts and substrates for graphene growth. The process includes three main stages: to diffuse the carbon on the metal film at high temperature; to deposit carbon on the metal surface while the decrease of carbon solubility during cooling; to form a graphene layer on the metal surface. The ideal way to synthesize graphene is to grow directly on the surface of materials, such as fibers and fiber assemblies. Therefore, it is urgent to develop an effective way so that the graphene grown on the metal surface will not produce 
defects or degrade when transferred onto non-metallic substrate surfaces. The most effective transfer method is as follows: firstly, polymethyl methacrylate (PMMA) or polydimethylsiloxane (PDMS) was used as a support layer under the metal layer where graphene could grow, and then the metal layer was removed by using an etchant, then the graphene could transfer onto the surface of support layer. If it is necessary the support layer could be removed by solvents, at last the graphene was transferred to the final target substrates. Figure $1 a, b, c$ shows the schematics of the process of transferring the graphene to PET substrates. CVD method can obtain high quality graphene with few or even single layer. Bae et al. obtained single-layer graphene sheets with resistance of $125 \Omega \mathrm{sq}^{-1}$ and light transmittance of $97.4 \%$ (wavelength of $500 \mathrm{~nm}$ ) by roll-to-roll transfer method [36]. However, the complicated process and high cost of CVD method limits its large scale production.

\subsection{Redox method}

As shown in Fig. 1d, the graphite was oxidized by strong acids and oxidants, the oxidized groups were inserted between the graphite layers, and the graphite layers were exfoliated via sonication or mechanical force. Then the graphene oxide (GO) must be chemically reduced to reduced graphene oxide ( $\mathrm{rGO}$ ) using hydrazine or thermal reduction. Although the redox method is relatively simple and is feasible for large-scale production, electrical properties of the $\mathrm{rGO}$ is degraded due to structural damage during the oxidation. Bo et al. utilized reduced graphene oxide ( $\mathrm{rGO}$ ) to make a gas-sensing sensors of which resistance value is $10^{4}-10^{5}$ $\Omega \mathrm{sq}^{-1}$ [32], Such a large resistance will affect the sensitivity of the sensing and the transmission of sensing signals.

\subsection{LPE method}

There are three steps of the LPE method: (i) Intercalation of Graphite with a chemical agent; (ii) Exfoliation of the intercalated graphite layers via sonication, (iii) Purification of the exfoliated flakes from un-exfoliated flakes via ultracentrifugation. The LPE method also can be divided into three types: chemical exfoliation (CE), sonication exfoliation (SE) and electrochemical exfoliation (EE). The CE and SE method are two different approaches, however, they often worked together. Graphene obtained from different LPE method had different size of flake, as shown in Fig. 2.

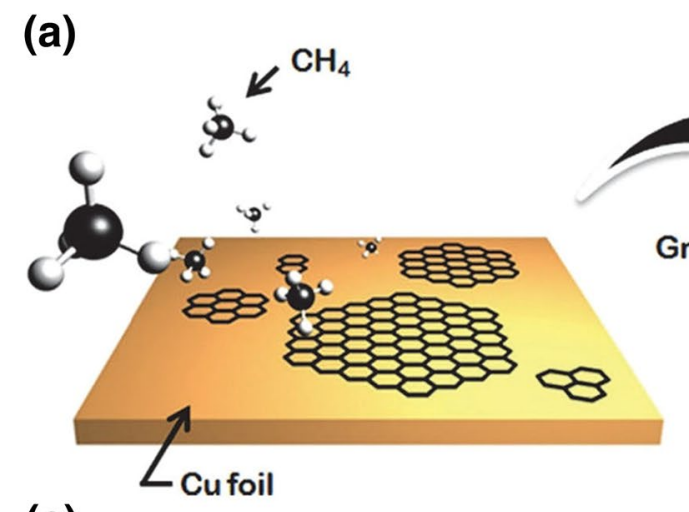

(c)

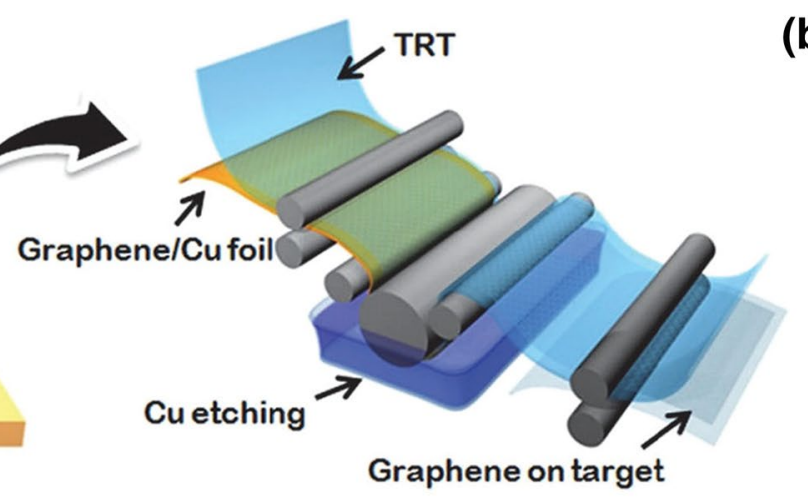

(b)

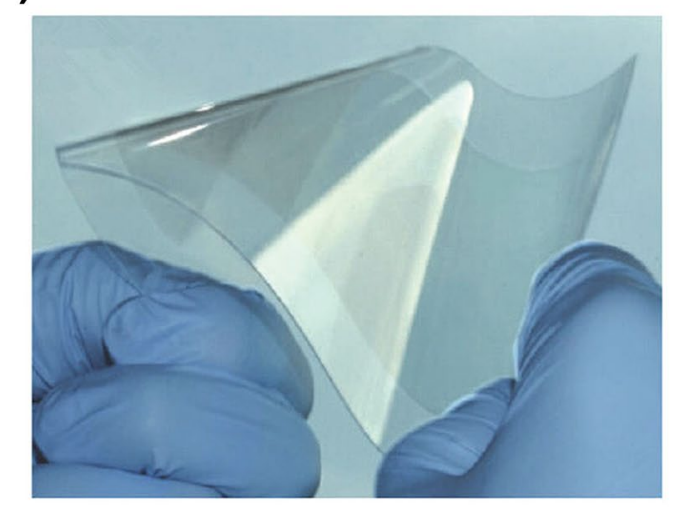

(d)

Fig. 1 a Schematics of the synthesis mechanism of CVD graphene on Cu foil. $\mathbf{b}$ The roll-to-roll transfer process of graphene to PET substrate. c Optical image of graphene on a flexible PET substrate. $\mathbf{d}$ Schematic illustration of the Redox process of graphene flakes generated from graphite [37] 

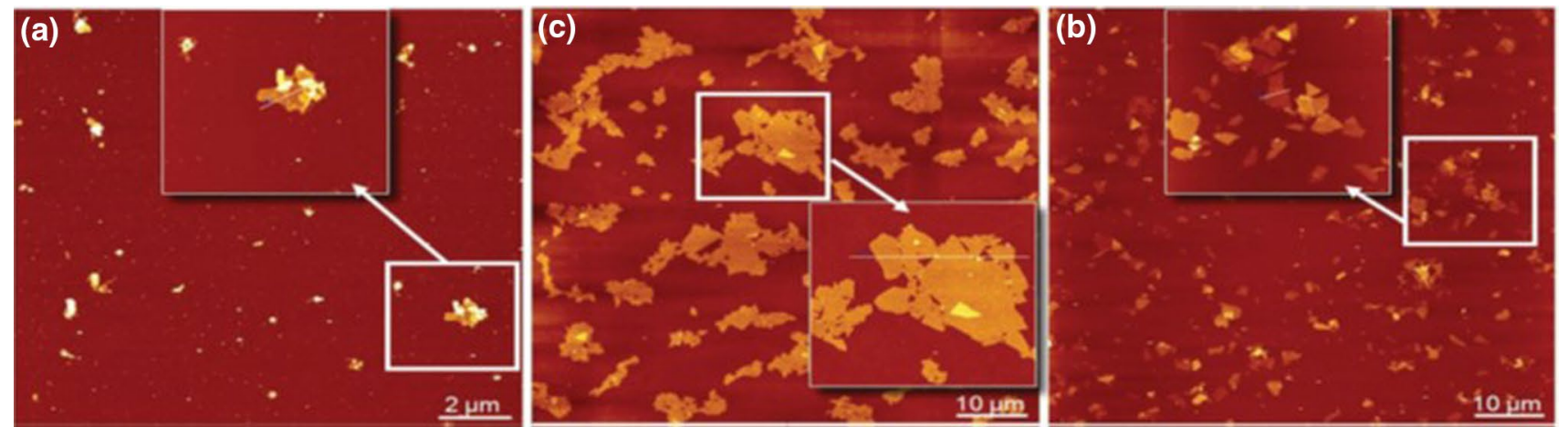

Fig. 2 AFM image of soluble flakes obtained by a sonication-assisted exfoliation-SE, b chemical exfoliation-CE and c electrochemical exfoliation-EE, the insets show a zoom-in taken of the white square areas [35]

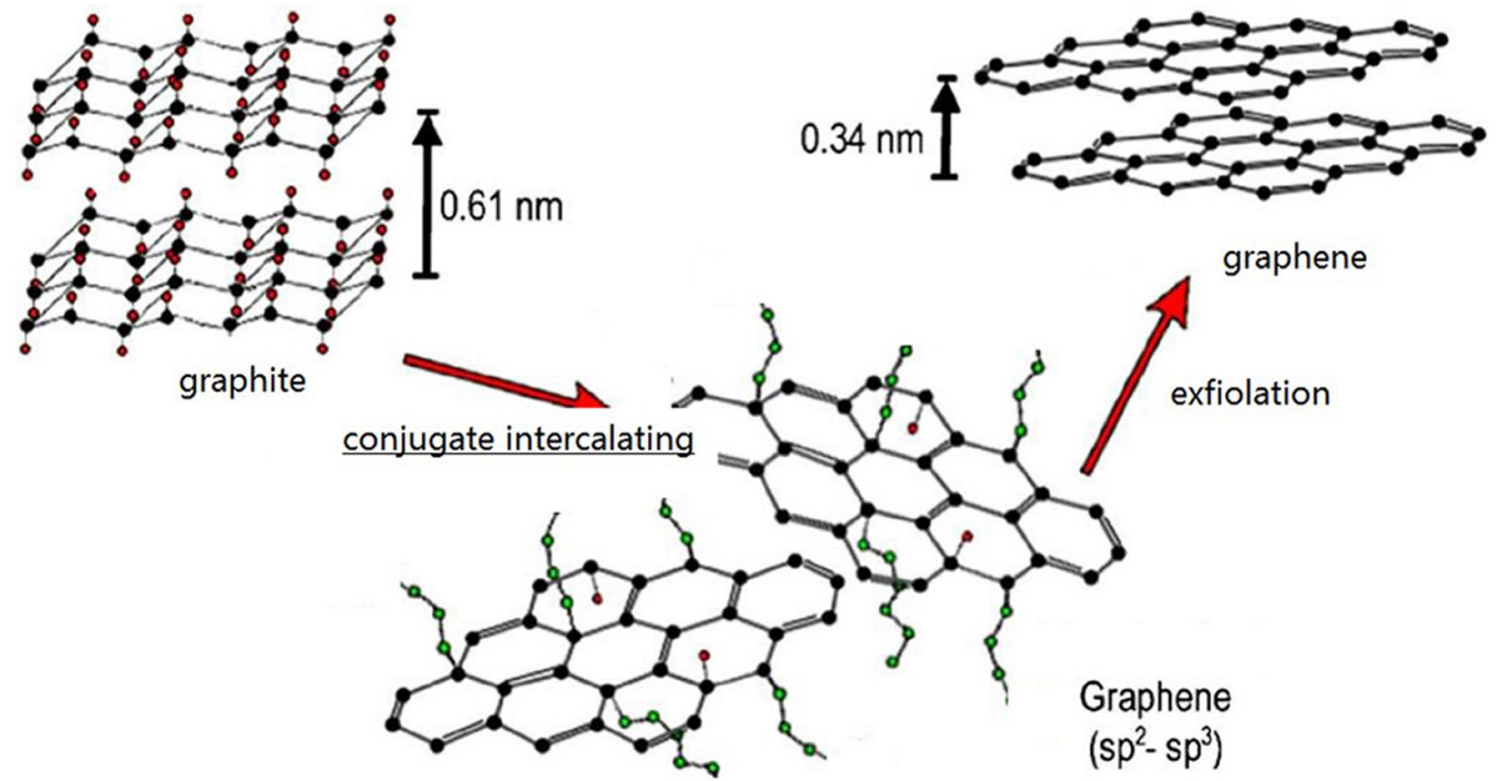

Fig. 3 Schematic of chemical exfoliation: chemical reagents intercalate graphite firstly, and then form a $\pi-\pi$ conjugation effect with the carbon atoms of graphene to prevent re-aggregation of the layers, and assisted with ultrasonic treatment to exfoliate graphene with few layers

As shown in Fig. 3, CE is a simple method to prepare the graphene without structural defects caused by redox, its uses chemical reagents that can $\pi-\pi$ conjugate with graphene to first intercalate the graphite and then exfoliate it to the graphene. The graphene obtained by CE method can be dispersed in common solvents(e.g. ethanol, acetone.) to form graphene solution (inks) which can be easily combined with other polymer solvents by casting or filtration [38]. However, the graphene flakes obtained by CE are usually with a relatively large size $(<10 \mu \mathrm{m})$ and the surface of the flakes are partially modified during the chemical solvent intercalation process, which cause some limitations on processing composite materials [35]. The SE method is the simplest and feasible method for large-scale production. Graphene can be obtained directly by putting graphite into solvent with ultrasonic treatment. Some dipolar aprotic solvents (e.g. $\mathrm{N}, \mathrm{N}^{\prime}$-dimethylformamide (DMF), $\mathrm{N}$-methylpyrrolidone (NMP), aqueous surfactant solution) are effective graphene dispersants. After ultrasonic treatment, graphene can form a relatively stable dispersant in the above solvent. Although the mechanism of ultrasonic dispersion is still not clear, the graphene nanosheets with the size below $1 \mu \mathrm{m}$ can be obtained. In addition, there are challenges in choosing a safe solvent that can reduce the number of graphene sheets and increase production $[39,40]$. Graphene obtained by EE method is more controllable and less destructive. As shown in Fig. 4, by adjusting the driving potential in a suitable electrolyte, the graphite electrode can form a graphene intercalation compounds (GICs) by positive and negative interphase intercalation, then exfoliated directly 
Fig. 4 Schematic of graphene exfoliated by graphite anode. In the water, graphite after electrochemical treatment $(10-20 \mathrm{v})$ make a suspension with colloids of chemically modified graphene that produced in imidazolyl ionic liquids [41]

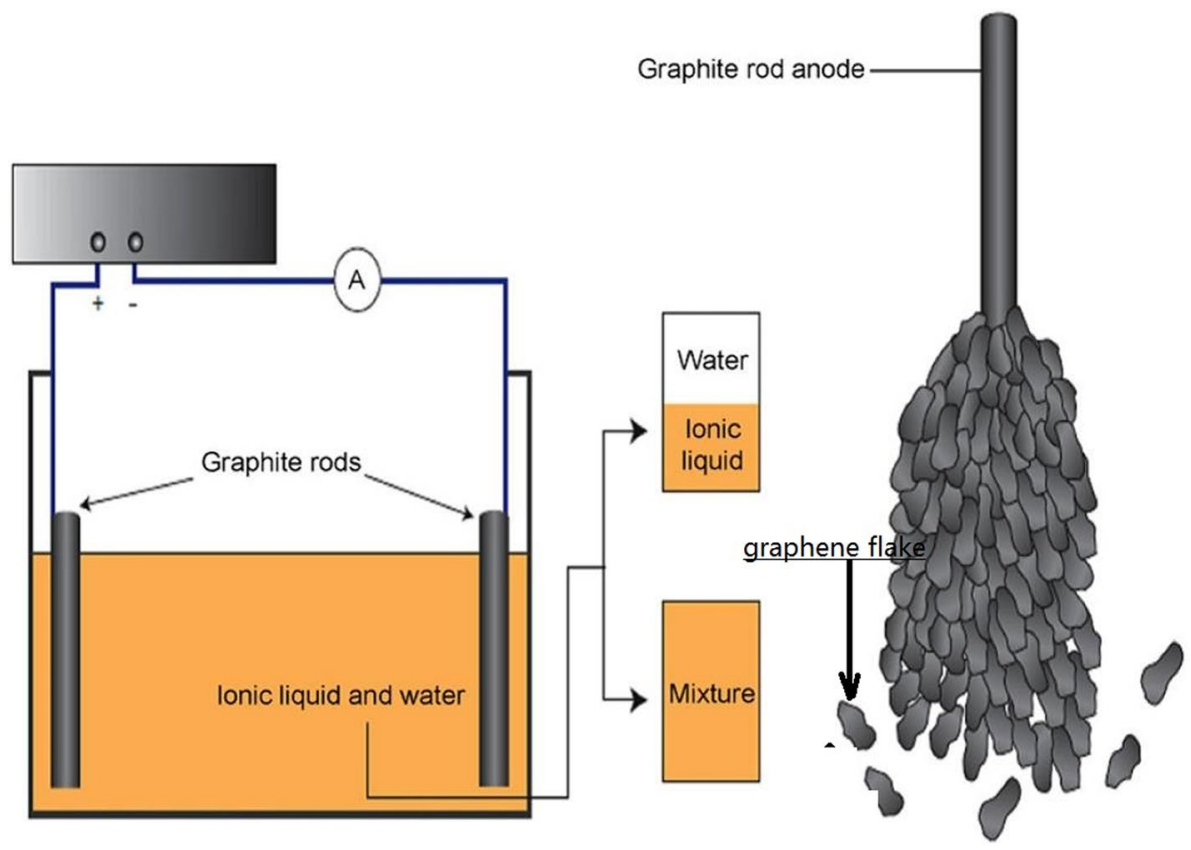

by solvolysis during electrochemical treatment or further heat treatment to form graphene platelets [41]. Although the size of graphene flakes can be adjusted $(1-10 \mu \mathrm{m})$ by the voltage of the electrode, the properties of graphene obtained by EE method are worse than CVD method and the large-scale production is still not achieved till now.

In summary, CVD method is able to grow large-area and high-quality graphene films on metal surfaces by gaseous carbon sources. However, the complicated process and high cost of CVD method limits its large scale production. Although the redox method is relatively simple and is feasible for large-scale production, electrical properties of the $\mathrm{rGO}$ is degraded due to structural damage during the oxidation. And the graphene obtained from different LPE method had different size of flake, $C E$ is a simple method to prepare the graphene without structural defects caused by redox, but its graphene flakes are usually with a relatively large size; SE is the simplest and feasible method for large-scale production, and the graphene nanosheets with the size below $1 \mu \mathrm{m}$ can be obtained; Graphene obtained by EE method is more controllable and less destructive, and the size of graphene flakes can be adjusted (1-10 $\mu \mathrm{m})$. However, the properties of graphene obtained by $\mathrm{EE}$ method are worse than CVD method and the large-scale production is still not achieved till now.

\section{Preparation of FGS}

Considering the cost and quality of graphene, as well as the interface properties between graphene and other fiber-forming polymers, in the past decade, many attempts had been done on the fabrication of the FGS. There are two major categories: one is to fabricate graphene fiber (GBF) firstly, then to process it to fiber assemblies or to form graphene woven fabric (GWF) structure and to transfer it to the surface of other elastic substrate; the other is to coat, print, dip the graphene solution with off-the-shelf fibers assemblies motherboard. Although the former has better sensing performance, its complicated process (e.g. the preparation of graphene by CVD method, graphene fibers spinning process) and its low stretchability limited its development in application. While the latter one is simple and available to achieve large-scale production. However, the relatively low sensing sensitivity, low durability and mismatch of the interface between graphene and fibers had to be further improved.

\subsection{Preparation of the FGS from fiber to fiber assemblies}

For the preparation of flexible strain sensors based on graphene fibers, graphene fibers or graphene composite fibers with good electrical conductivity were first prepared and then further processed into fiber assemblies. Wet-spinning process is the main method to prepare the graphene or graphene composite fiber, as shown in Fig. 5 , the well dispersed graphene solution with liquid crystal state $(\mathrm{LC})$ can be aligned along the axial direction by external force intervention and eventually coagulated into a fiber [42]. In the wet spinning process, the GO was first dispersed in an aqueous solution to prepare GO slurry with a certain viscosity, and the slurry was sprayed from the spinneret through a wet spinning device, and then injected 


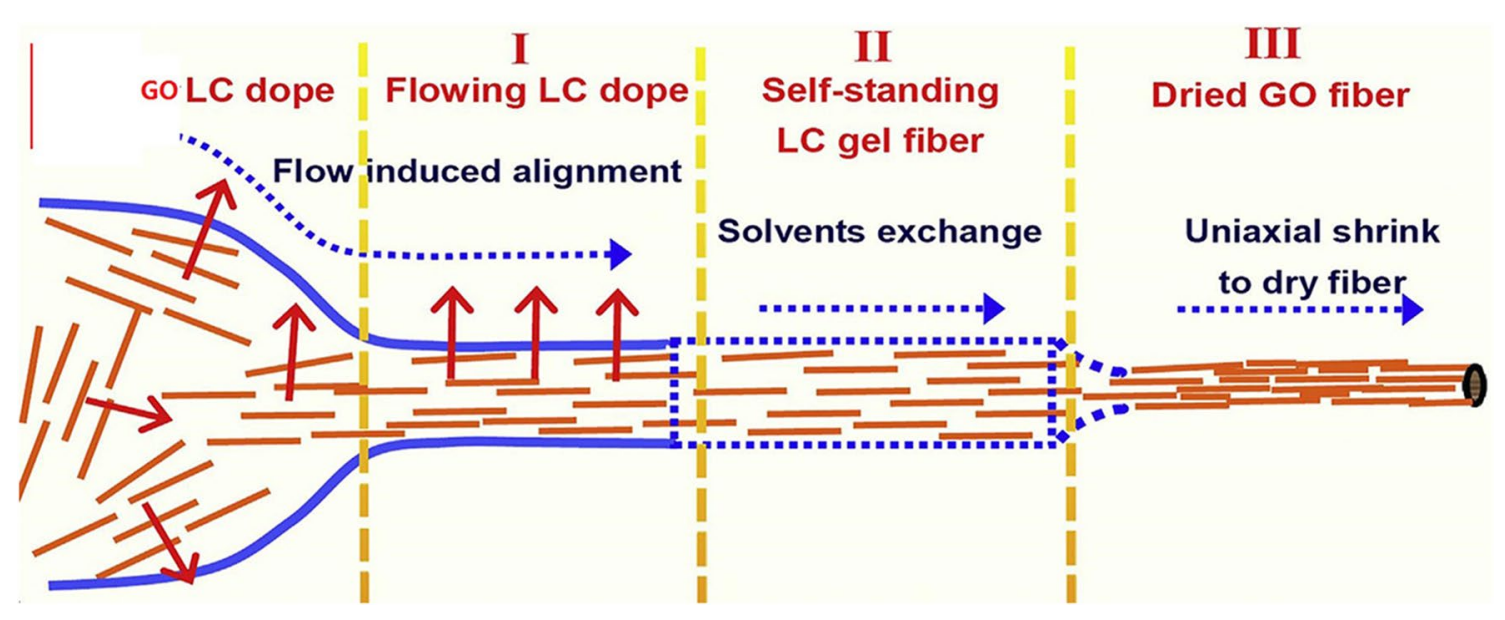

Fig. 5 Schematic of graphene oxide fiber formation. Spinning solution of graphene oxide with liquid crystal (LC) structure undergoes. (i) Directional flow induced orientation alignment (ii) the process of exchanging solvents process to form gel (iii) dried to GO fiber [42]

Fig. 6 Schematic of wet spinning device: processing graphene oxide fiber. a Rotary coagulation bath. b Rotary draft roller [51]
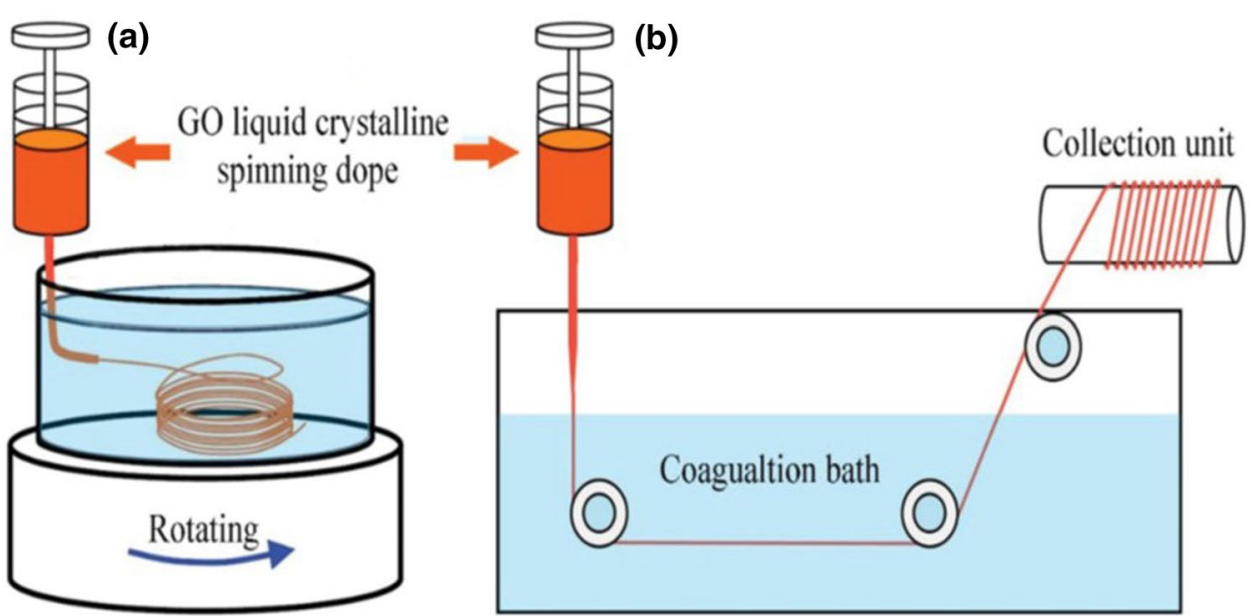

into the coagulation bath to form colloidal fibers. After a period of coagulation, the colloidal fibers were extracted through drawing orientation and dried to graphene oxide fiber (GOF), if required, it can also be further reduced to graphene fiber (GRF). To ensure the uniform continuity from gel state to fiber, maintaining a certain move speed of $\mathrm{GO}$ is necessary at the beginning of the gel process. Using a rotating bath or using a rotating roller can offer a certain draft to GOF from the gel to the fiber formation [43], as shown in Fig. 6a, b. The spinnability of the GOF or GRF produced by wet spinning is determined by the transverse dimension of the GO flakes and the concentration of the GO solution. Long and strong GOF or GRF can be spun only when the GO in the slurry is completely in the nematic phase [44]. In addition, the coagulation bath plays an important role in the processing process and the performance of GOF or GRF, because gel occurs when the coagulant broke the stable of GO. Non-solvent phase separation gel, acid-base imbalance gel, cross-linking induction gel, low temperature freeze drying can be used in coagulation bath [45]. Cong et al. prepared GOF with diameter of 25-120, strength of $145 \mathrm{MPa}$ and the Young's modulus of $4.5 \mathrm{GPa}$, after reduced to GRF, its the strength and the Young's modulus increased to $187 \mathrm{MPa}$, 8.7 GPa, at the same time, the electric conductivity of the GRF was $35 \mathrm{~S} \mathrm{~cm}^{-1}$ which can be used as sensing materials [46]. Shear stress was introduced to wet spinning, the ribbonlike GRF prepared by this method has high flexibility and exhibits good performance in many applications such as flexible strain sensors or counter electrodes [47]. Figure 7 is the SEM image of the axial and cross-section of GOF. By adjusting some parameters during the wet spinning process, GOF or GRF could be prepared with a special section and good electrical properties. The GO and carboxymethyl cellulose spinning slurry was injected into the gel bath by the inner and outer two coaxial micro-spinneret simultaneously, which formed a graphene/carboxymethyl cellulose composite fiber with skin-core structure (Fig. 8). 
Fig. 7 SEM image of GOF prepared by wet spinning a axial, b cross section [51]
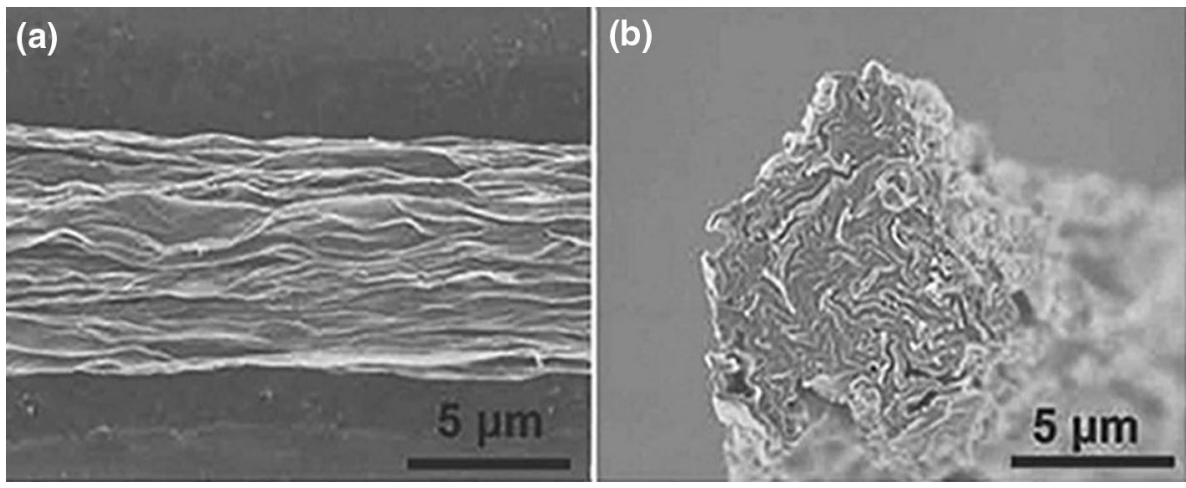

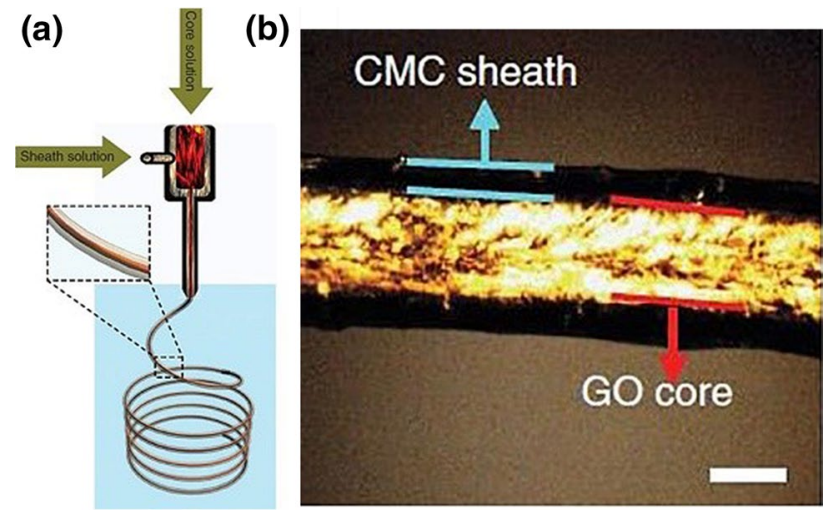

Fig. 8 Schematic of GOF with sheath-core structure: graphene oxide and carboxymethyl cellulose spinning slurry injected simultaneously from the two spinneret (left), GO/carboxymethyl cellulose composite fiber with sheath-core structure (right).[48]
The carboxymethyl on sheath layer has the characteristic of ionic conduction while the graphene on core layer has the characteristic of electronic conduction. This kind of skin-core structure fibers could be used as a capacitive sensor [48]. Hollow GOF as well as GOF multifilament containing 50 graphene oxide microfiber were also prepared by using the wet spinning with multi-spinneret $[49,50]$. These GOF further reduced to the GRF with good electrical properties which could be used as strain sensors.

Another method to prepare GRF is dry forming. As shown in Fig. 9, GO dispersion solution was sealed in glass tube or polypropylene tube and precipitated to gel by heating or chemical reduction at a high temperature, GRF can be obtained by further removing the solvent (one end of tube is closed to retain heat, and the other end is open to release solvent). Generally, the high temperature
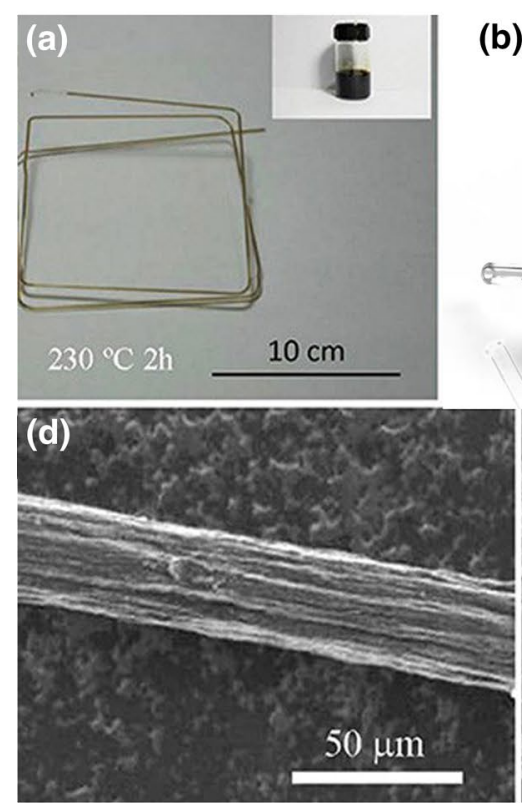
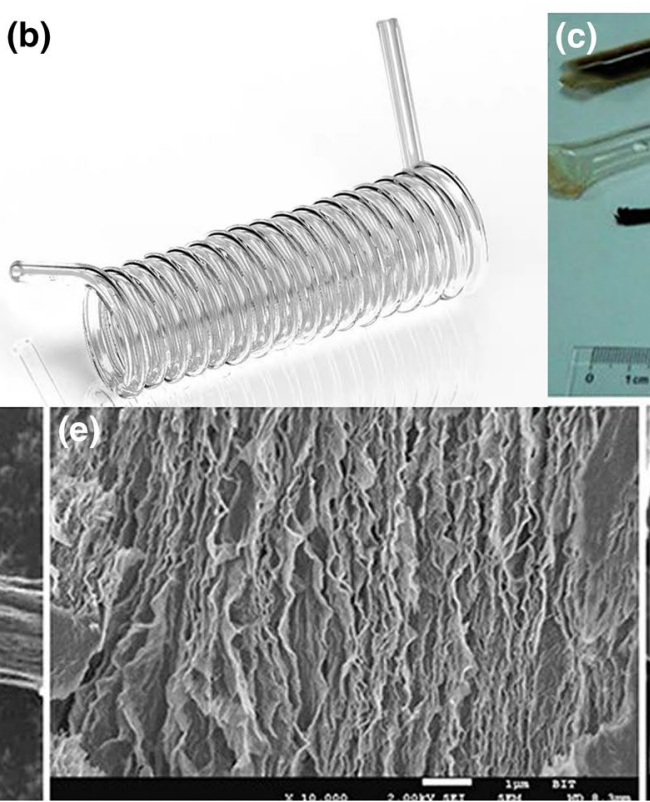
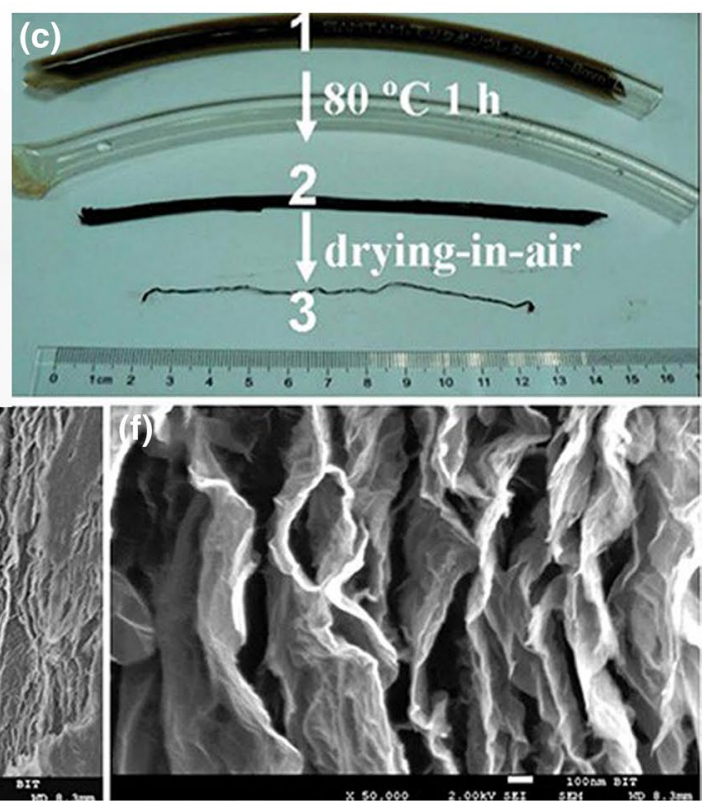

Fig. 9 Graphene fiber is dry-forming in a microtube a glass tube [52] The inset shows that the GO dispersion in a vial b the quartz capillary $\mathbf{c}$ PP pipe [53] (d) and e, $\mathbf{f}$ SEM images of the side and cross section of GBF [52] 

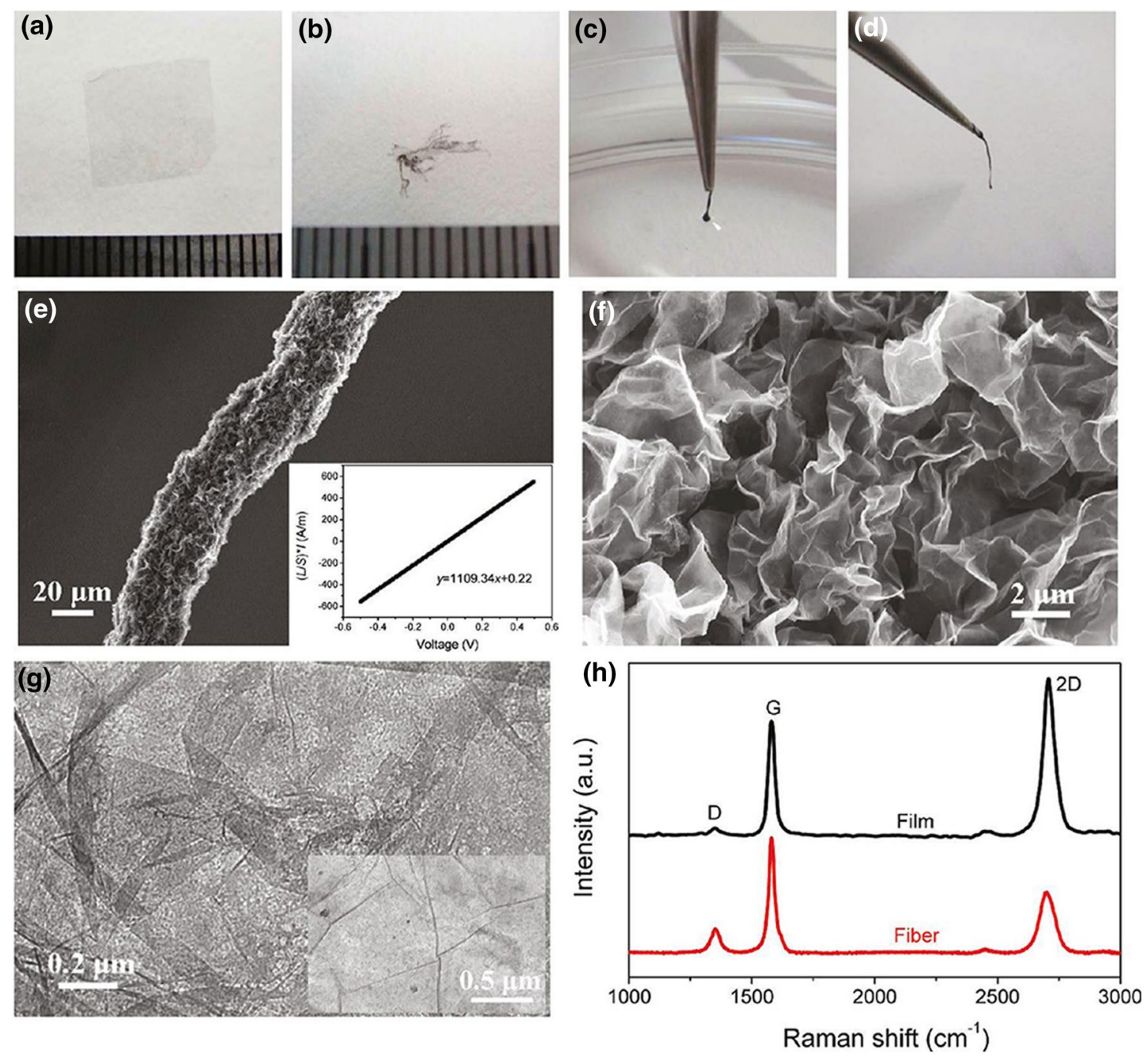

Fig. 10 Self-assembly from graphene film to fiber a Graphene film. b The graphene film was self-assembled into a fibrous structure. c Graphene fiber extracted from ethanol. d Porous wrinkled structure after drying. e Low-magnification SEM image of graphene fiber (inset is current density-voltage curve, electric conductivity

processing can not only remove the solvent, but also thermally reduces the $\mathrm{GO}$ to $\mathrm{RGO}$. Dong et.al encapsulated $\mathrm{GO}$ solution in a fine glass tube and treated with $230{ }^{\circ} \mathrm{C}$ to remove solvents and thermally reduced GO to RGO, the prepared GRF exhibited good electrical conductivity of 10 $\mathrm{S} \mathrm{cm}^{-1}$ [52]. However, two disadvantages of dry forming method were that the size of GRF was limited to the size of the encapsulated tube and the solvent removal was difficult, thus it was not suitable for large scale production.

Graphene can also be processed into a fiber shape using its self-assembly properties. Li et al. assembled graphene film obtained by the CVD method into a graphene fiber through self-assembly method [54]. As shown in Fig. 10, after removing from a copper foil, the grapheme

is $1000 \mathrm{~S} \mathrm{~cm}^{-1}$ ). $f$ SEM and TEM image of graphene fiber, showing plicated and porous structure (inset is the transmission electron microscopy (TEM) image of original graphene film). $\mathbf{h}$ Raman spectroscopy of graphene film and fiber [54]

film was transferred to an organic solvent (e.g. ethanol), the film first floats on the surface of the solvent (Fig. 10a) and then the edge of the film rolled up immediately, at last the film rolled into a fiber and sank into the solvent (Fig. 10b). Tweezers were used to remove the fibrous film from the solvent (Fig. 10c), with the evaporation of ethanol, the film was shrunk into GRF with multi-porous structure. Although the GRF obtained by self-assembly method with the conductivity of $1000 \mathrm{~S} \mathrm{~cm}^{-1}$ was promising for sensing materials, its low strength and difficulty to be further process into a fiber assemblies structure limited its practical application.

In addition, graphene composite fiber (GCRF) can be prepared using wet spinning method by mixing the fiber 
forming polymer solutions (e.g. polyvinyl alcohol, cellulose, polyacrylonitrile) with graphene or graphene oxide solution. With a low ratio of graphene or graphene oxide, the mechanical properties of the GCRF were improved significantly, but it did not have the electrical conductivity. If the graphene was used as the main component to combine with other fiber forming polymer, the excellent electrical conductivity of GCRF was retained and its mechanical properties were also improved. The electrical conductivity properties of GCRF can be further enhanced by adding other conductive nanofillers(e.g. silver nanowires [55], carbon nanotubes [56], polypyrrole [10], polyaniline [57]). Kou et al. used polyvinyl alcohol /graphene oxide mixed solution to prepare GCRF, due to hydrogen bonding, PVA was well combined with graphene oxide, which can be processed into GOF by wet spinning method and further reduce to GCRF (Fig. 11). By adding 5 wt\% PVA, the fiber exhibited good mechanical properties (stress of $200 \mathrm{MPa}$ ) as well as good electrical conductivity $\left(350 \mathrm{~S} \mathrm{~cm}^{-1}\right)$ [58]. $\mathrm{Xu}$ et al. covered the silver nano wires on the surface of $\mathrm{GO}$, and reduced the $\mathrm{GO}$ to $\mathrm{RGO}$ by vitamin C, then prepared graphene/silver nanowire composite fiber by wet (a)

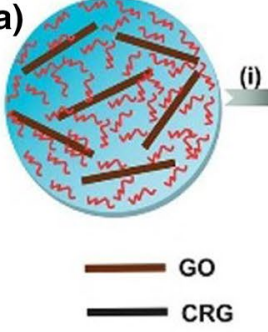

(b)

b)

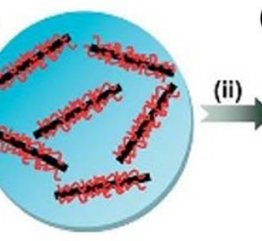

m PVA

miaten CRG@PVA

(c)

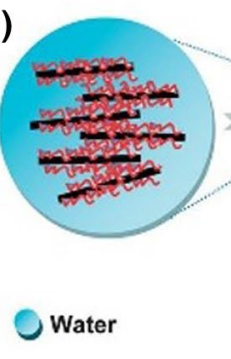

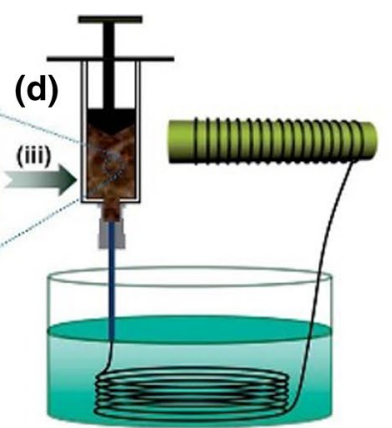

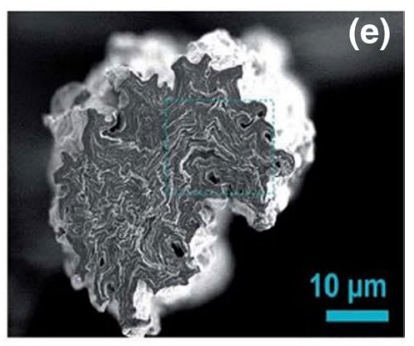

Fig. 11 Schematic of the preparation process of graphene/PVA composite fiber (a) PVA coated on the surface of graphene oxide. b Reduced to graphene with hydrazine hydrate, c PVA-coated gra-

phene liquid crystal after oriented. d Wet spinning e Fiber profile, covered with PVA in the surface [58] (a)

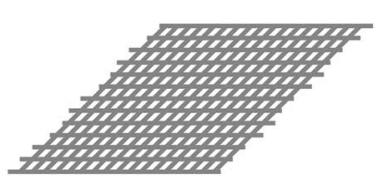

Copper mesh (b)

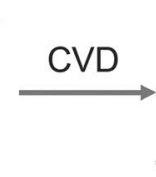

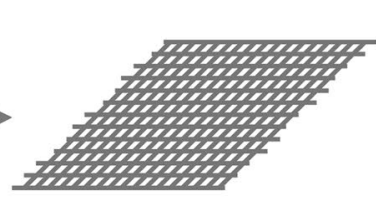

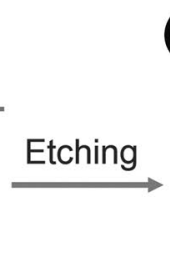

Graphene growth (c)

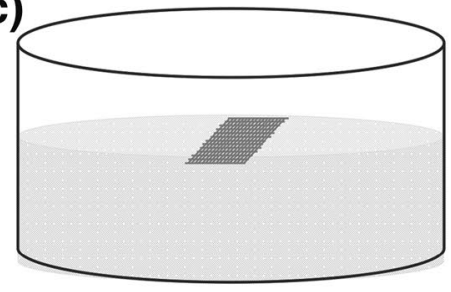

$\mathrm{FeCl}_{3} / \mathrm{HCl}$ solution

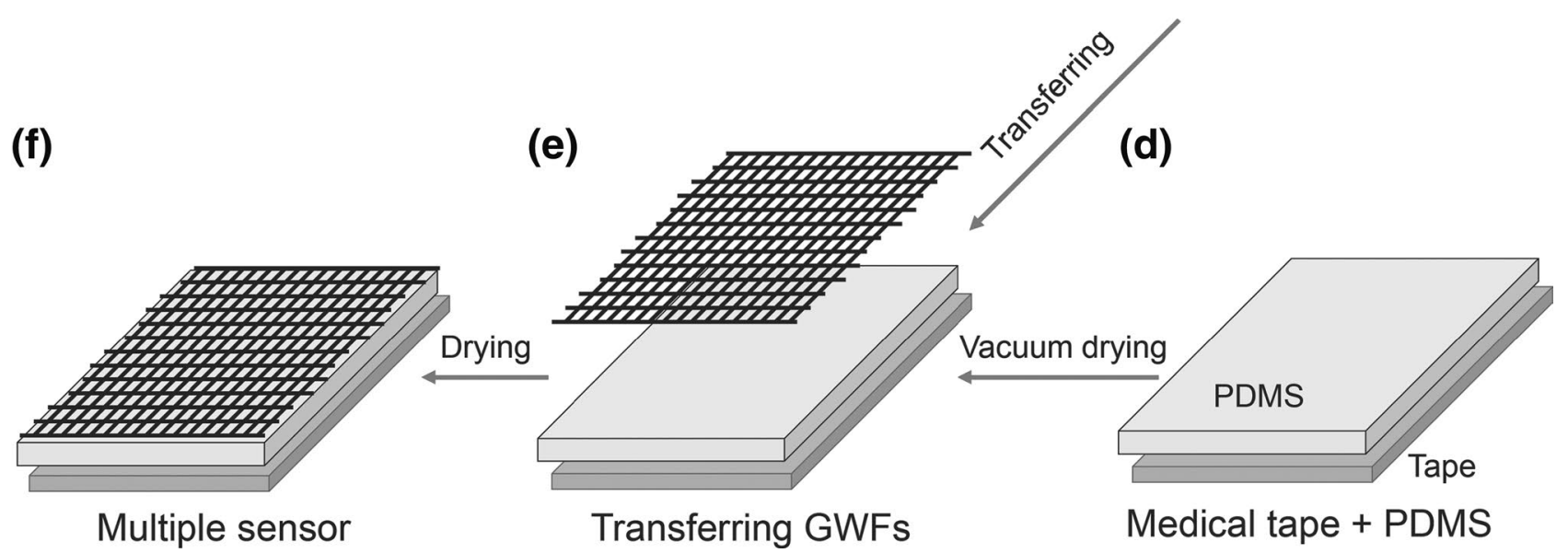

Fig. 12 Schematic illustrations of fabrication procedure of FGS based on GWF-PDMS-medical tape film [59] 


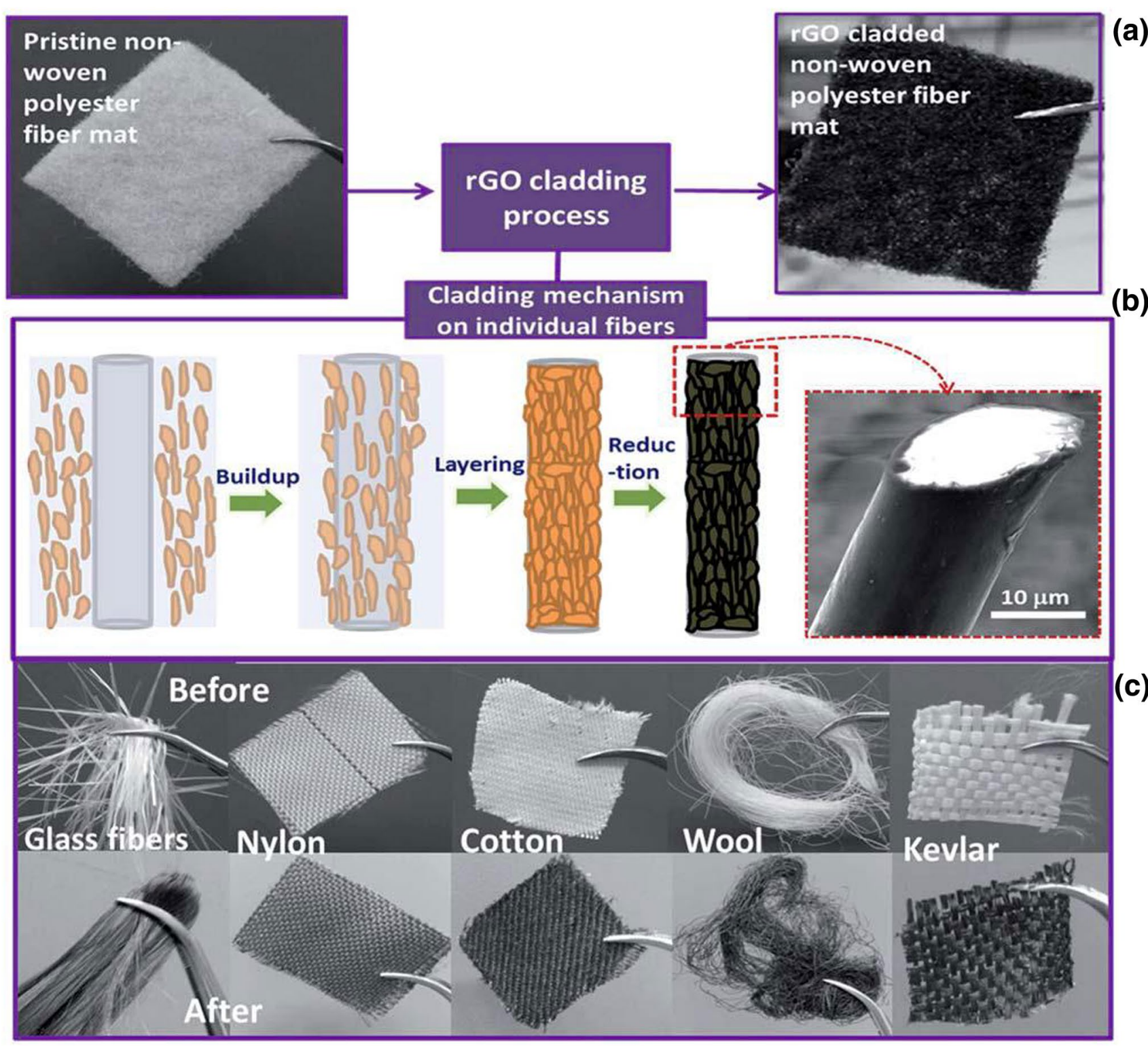

Fig. 13 Preparation of FGS utilizing existing fiber assemblies structure. a Non-woven fabric surface covered with redox grapheme. $\mathbf{b}$ Yarn surface covered with graphene oxide and then reduced to grapheme. $\mathbf{c}$ Various fiber assemblies are covered with graphene [60]

spinning. The GCRF exhibited good electrical conductivity $\left(9.3 \times 10^{4} \mathrm{~S} \mathrm{~cm}^{-1}\right)$ and mechanical properties to be used as sensors material [51].

The FGS can also be fabricated by graphene woven fabric (GWF) method. Firstly the graphene is growing on a metallic mesh (normally $\mathrm{Cu}$ ) by CVD method, then the $\mathrm{Cu}$ mesh is removed by $\mathrm{FeCl}_{3} / \mathrm{HCl}$ treatment and the resulting graphene fiber with the woven like structure called GWF is transferred to other elastic substrate and further encapsulated into FGS. Although time-consuming and complex processes, the FGS prepared by this method with high sensitivity due to good sensing properties of graphene obtained by CVD, can be used to detect the strain in very small value. Wang et al. prepared a FGS with ultra-sensitive of GF of 1000 under $2-6 \%$ strains, $10^{6}$ under higher strains (> 7\%), and 35 under small strain of $0.2 \%$. (Fig. 12) [59].

\subsection{Preparation of the FGS based on off-the-shelf fiber assemblies}

As shown in Fig. 13, the ordinary fiber assemblies, such as fabrics (e.g. woven fabric, knitting fabric, non-woven fabric) and yarns, are usually used as the motherboard 
Fig. 14 Schematic of preparing FGS by dip-coating method (a) coating graphene on the surface of existing yarn by LBL method (b) yarn structure after coating [1]

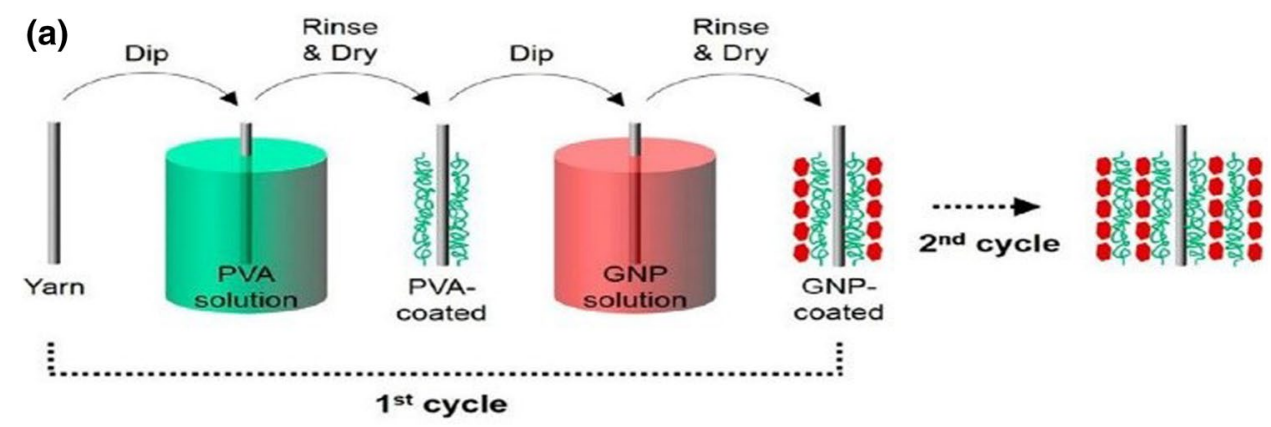

(b)

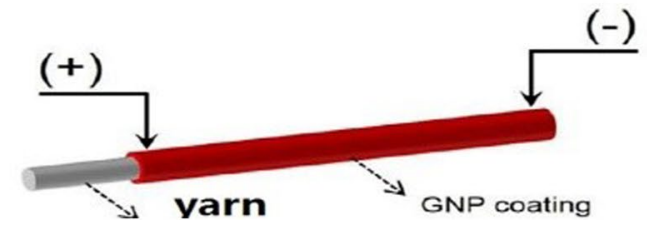

substrates to fabricate FGS by integrating the graphene on the surface of this substrates through dip-coating, printing or filtrating.

The dip-coating is a simple and most commonly process by dipping the fiber assemblies with $\mathrm{GO}$ or graphene solution for a period of time and then dried. The fiber assemblies coated with graphene or GO (if it is necessary, the $\mathrm{GO}$ can be reduced to $\mathrm{rGO}$ ) can be used as a FGS. Park et al. reported a highly stretchable (strain $>70 \%$ ), high sensitive (GF > 100) FGS by layer-by-layer (LbL) dip-coating graphene solution on the surface of yarn substrates [1] (Fig. 14). Du et al. also reported a FGS by dip-coating the
GO on non-woven fabric substrate and then reduce $\mathrm{GO}$ to rGO by using hydroiodic acid, the prepared FGS exhibited good sensitivity to strain (GF of 7.1) and good piezoresistivity $\left(\mathrm{K}=0.057 \mathrm{kPa}^{-1}\right)$ [61].

Vacuum Filtration method can also be used to coat GO or grapheme solution on fiber assemblies with high density. Yin et al. firstly coated GO on a high density bandage fabric made of cotton fibers by vacuum filtration and then pyrolysis reduced GO to $\mathrm{rGO}$ by the flame of ethanol (Fig. 15), the prepared FGS exhibited large stretchability (57\%) and good sensing performance (GF > 416) [62].
Fig. 15 a Schematic of the entire procedure for the fabrication of FGS based on woven fabric. b SEM micrographics of FGS magnification: $40 \times, 200 \times$, $3000 \times$, and $10000 \times .[62]$ (a)

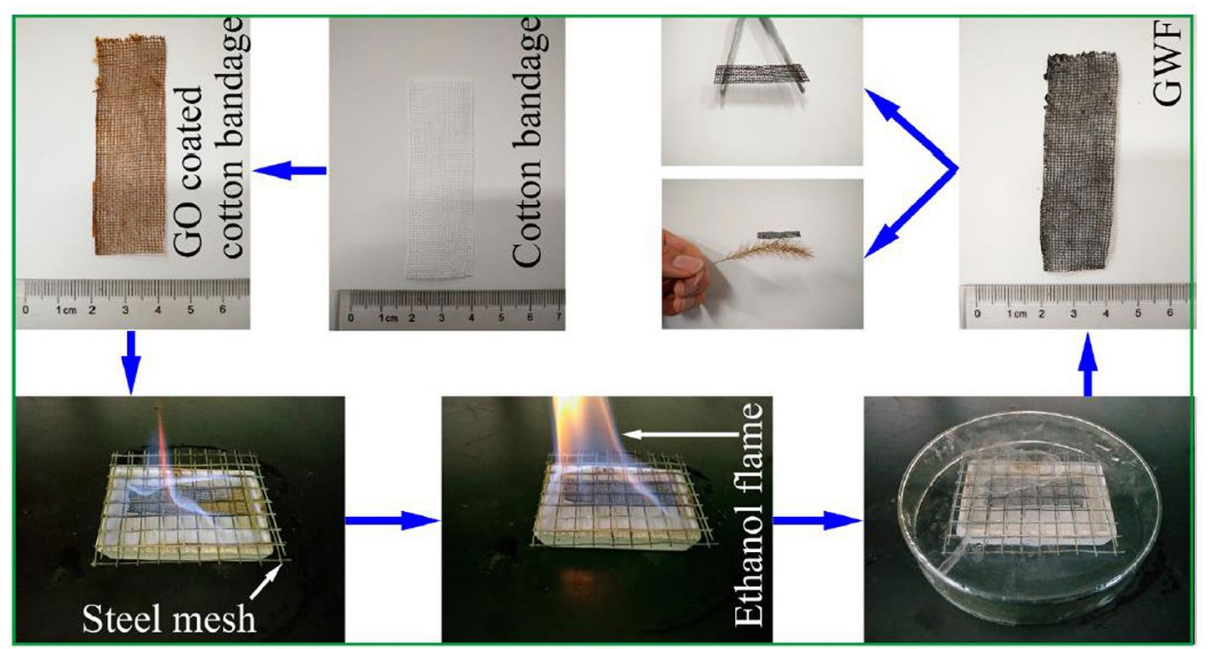

(b)
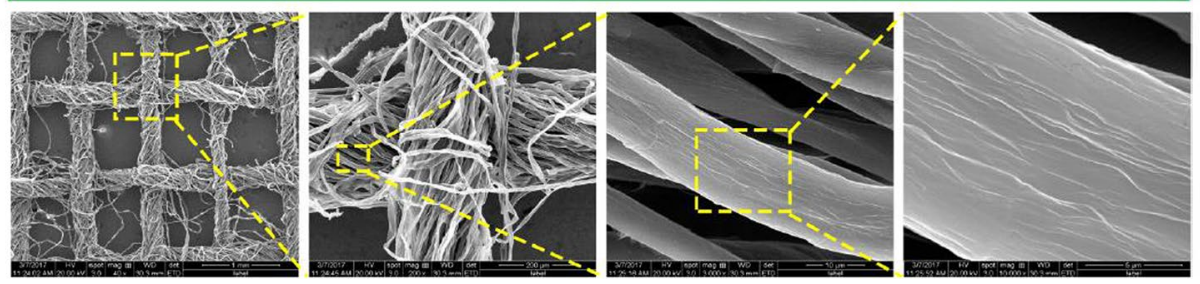

SN Applied Sciences a SPRINGer Nature journal 
4 .

Compared with dipping-coating and vacuum filtration method, ink-jet printing could precisely deposite the grapheme on the specific area of the fiber assemblies, thus raw materials and energy could be saved [63]. However, it is a challenge to coat a continuous conductive path on the surface of the fiber assemblies due to its rough and porous surface structure. Karim et al. developed a printing method to coat graphene on woven fabric (Fig. 16), firstly the polyester/cotton woven fabric was pretreated by spraying a layer of $200 \mu \mathrm{m}$ thick styrene/divinyl benzene emulsion as a flat substrate, then $\mathrm{GO}$ was coated on the flat surface of fabric by printing, at last GO was reduced to rGO by vitamin C. The prepared FGS with good electrical properties (resistance of $1.18 \Omega \mathrm{sq}^{-1}$ ) can be used to capture the heart rate [64].

\section{Sensing mechanism}

Strain sensors have different sensing mechanisms because of the types of materials, micro/nanostructures, and fabrication process. According to the FGS, there are three mainly sensing mechanism: geometric effect caused by the structure or dimension changes of fiber assemblies, the piezoresistive effect caused by the essential characteristics of graphene, and the connection-disconnection effect caused by sliding of grapheme sheets [65].

\subsection{Geometric effect}

When materials are stretched, they tend to shrink in transverse direction and elongate in length direction which based on the Poisson's ratio of $U$. The resistance is given by $R=\rho L / A$, (where $\rho$ is the electrical resistivity, $L$ is the length, and $A$ is the cross-sectional area of the conductor). Resistance of a conductor increases with the elongation of its length and the shrinkage of its cross-sectional. Different from other sensors, the changes in resistance of the FGS are first caused by structural changes of fiber assemblies, and then caused by changes in the length and transverse dimensions of the material itself, so the geometric effects are more complicated. Ma et al. researched the relationship between network structure of yarn assemblies and electromechanical properties of knitted or weave fabrics, the resistance of FGS based on fabric will change firstly

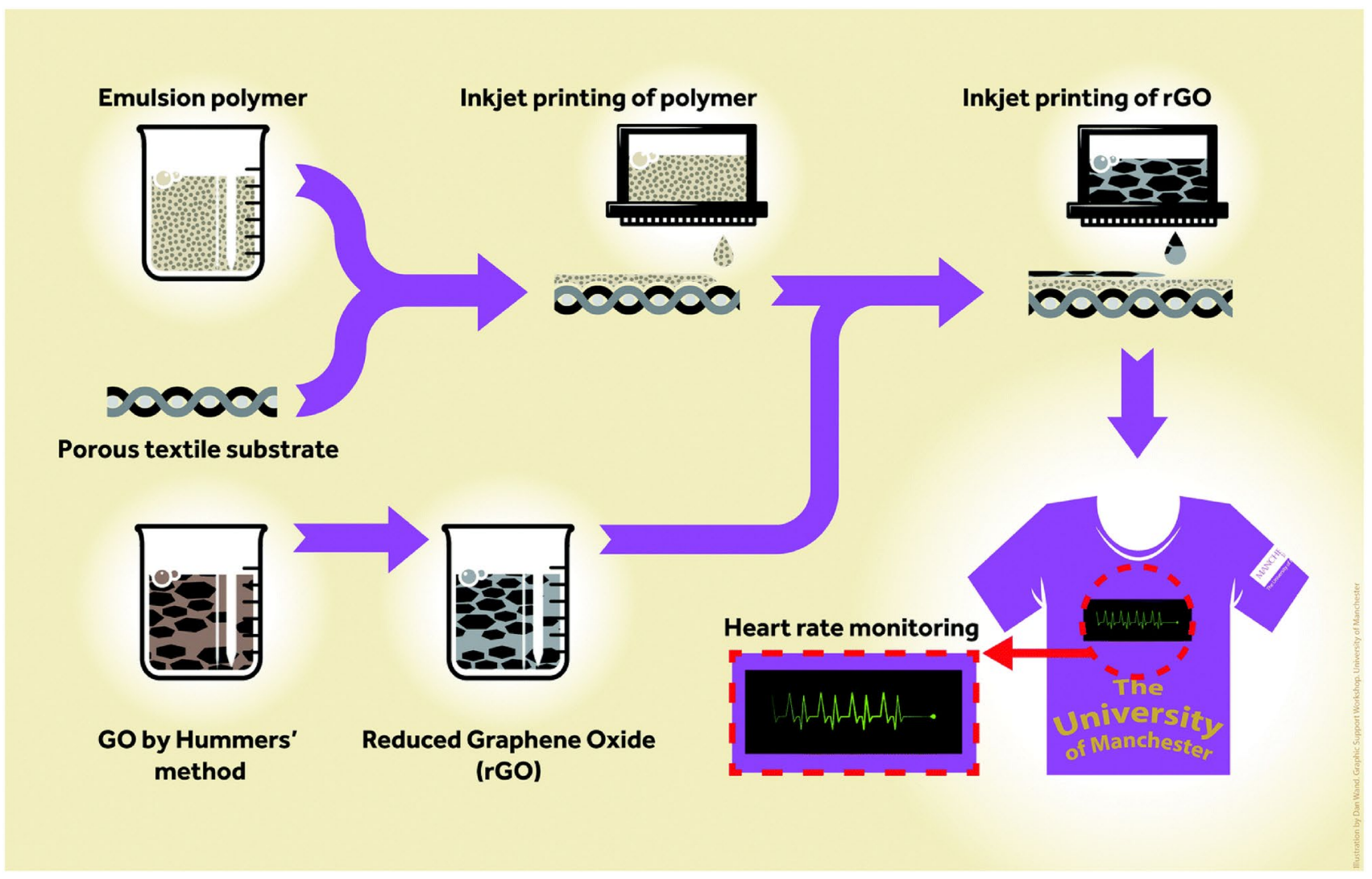

Fig. 16 Schematic of the preparation of FGS by inkjet printing method [64] 
by changes of fabric structure, then by the deformation of yarn when the yarn was stretched from curve to fully stretch [66].

\subsection{Piezoresistive effect}

Change in the resistance of materials caused by the structural deformations and density variation is known as piezoresistivity. The relative resistance change in this case can be written as: $\Delta R / R=(1+2 v) \varepsilon+\Delta \rho / \rho$, where $u$ is the Poisson's ratio of the fiber material, $\varepsilon$ is the strain, and $\Delta \rho$ is the density change value under force, $\rho$ is the initial density. The former indicates the influence of the geometric effect, and the latter indicates the piezoresistive property of the material, that is, the change in resistance caused by the density of material under pressure [65, 67]. In the case of large strain, the geometric effect plays important role in sensing mechanism and the piezoresistive effect can be negligible, while Although the materials as graphene has good piezoresistive effect and with high sensitivity, the strain requirement of skin-mountable and wearable strain sensors ( $\varepsilon>30 \%$ ) far exceeds the measurement limit of graphene itself. The stretchability of the fiber assembly can makes up for this shortcoming, which is also the advantage of the FGS. In the case of small strain, the piezoresistive effect plays important role in sensing mechanism $[68,69]$.

\subsection{Connection-disconnection effect}

Compared with other sensors, the resistance changes of the FGS caused by the connection-disconnection effect is often synergistic with the geometric effects. When graphene is well combined with fiber forming polymer, its excellent electrical conductivity provides a good electronic path through the connection between adjacent sheets. Some of the original connected graphene sheets lose overlapping areas and disconnected when stretching, thereby the electrical resistance increased. On the other hand, the overlapping areas and connection points of adjacent graphene sheets will increase when compressing, thereby the electrical resistance reduced. This is known as connection-disconnection effect. From the microscopic structure, the disconnection between the overlapping nano-conductive materials under stretching is due to that its weak interface with the stretchable polymer interface and large stiffness mismatch, resulting in the slippage of the nanomaterial $[62,70,71]$. Because the fiber and fiber assemblies have a certain strain recovery ability, on the situation of small strain, the disconnected graphene sheets will be back to its initial position when releasing the force, but on the situation of large strain, the disconnected graphene sheets will lose its initial position and lead to nonlinear resistance variation due to the residual strain or stiffness mismatch between graphene and fiber forming polymer. This is the characteristic of FGS that has the properties of high sensitivity and low linearity.

In summary, the challenges and perspectives based on the sensing mechanism can be summarized as follows: (i) to improve the dynamic mechanics of fibers forming polymer. (ii) to research the relationship between the structure of fiber assemblies and its electrical-mechanical properties. (iii) to enhance the interface properties between fibers forming polymer and graphene.

\section{Sensing performance indexes}

Strain sensing performance of FGS are characterized by performance parameters such as stretchability, sensitivity, linearity, hysteresis, response and recovery time, overshoot behavior and durability. These parameters are crucial to evaluate the performance of FGS and to determine its application field.

\subsection{Stretchability}

The stretchability is an important index which depends on nanomaterial structure of fiber assemblies, even the manufacturing process, and also influences its application. For example, a sensor with small strain and high sensitivity can be used to detect the pulse, heart beat, while a sensor with high stretchability (strain $>30 \%$ ) and with relatively low sensitivity can be used to detect the movement of limbs and joints $[72,73]$. Because GRF/GOF exhibits low stretchability $(\varepsilon \leq 10 \%)$ [74] (e.g. breaking elongation of GOF and GRF prepared by wet spinning is $6.8-10.1 \%$ and $5.8 \%$ [51], and breaking elongation of GRF prepared by dry-forming is only $4.2 \%$ ) [52], and FGS based on fiber exhibited low stretchability, so it is necessary to further process the graphene fiber to fiber assemblies structure or to integrate like GWF structure with other elastic polymers substrate ( e.g. PET, PA66, PU) $[10,75,76]$. On the other hand, the FGS based on off-theshelf fiber assemblies normally has higher stretchability, especially on knitting fabric [77].

\subsection{Sensitivity}

Sensitivity (GF) is generally expressed as the ratio between the relative change in the electrical signal (usually resistance or capacitance) and the applied strain (Fig. 17a.), that is $\mathrm{GF}=(\Delta \mathrm{R} / \mathrm{RO}) / \varepsilon$ or $\mathrm{GFs}=(\Delta \mathrm{C} / \mathrm{CO}) / \varepsilon$ [78]. For 
Fig. 17 a Different sensitivity of sensor [96]. b Nonlinear response of sensor [82]. c High linear response of sensor [86]. d Hysteresis during stretch and release cycles [87]
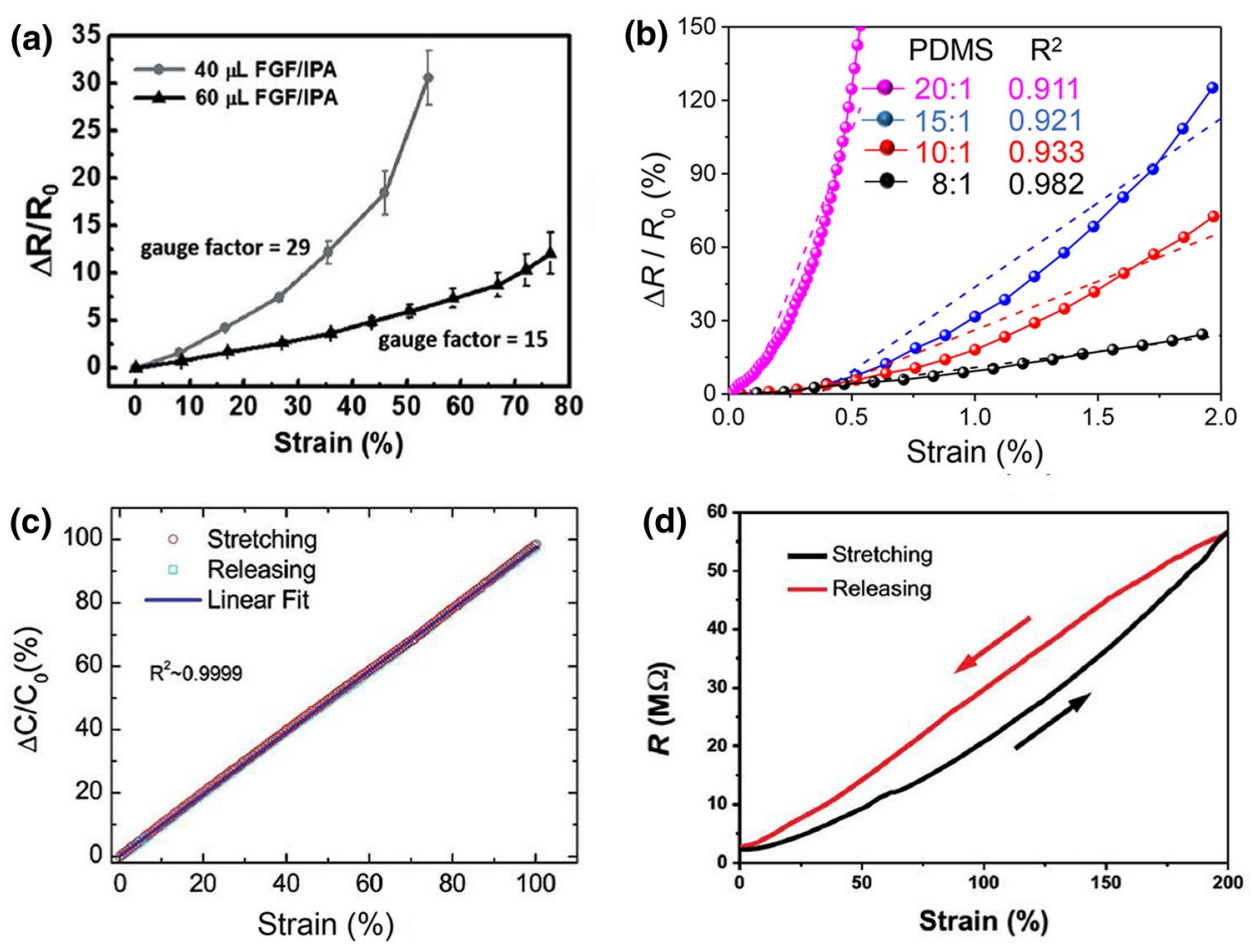

piezoresistive sensors, since the strain caused by pressure is not easy to measure, it can also be expressed by the ratio between the change of resistance (capacitance) and the given pressure, $\mathrm{S}=(\Delta \mathrm{R} / \mathrm{R} 0) / \Delta \mathrm{P}$ or GFs $=(\Delta \mathrm{C} / \mathrm{CO}) / \Delta \mathrm{P}$ [79]. Sensitivity is also an important indicator of the FGS which i MPact on the accuracy of measurement. For precise measurement of small strains, such as pulse and heart beat, high sensitivity is necessary. Compared with the other sensor only on piezoresistive effect, the FGS is higher sensitive due to the synergy of the three mechanisms of geometric, connection-disconnection and piezoresistive effect $[10,80,81]$. By using the high quality graphene obtained by CVD method can further improve the sensitivity of FGS. Wang et al. fabricated a FGS by CVD growing graphene on GWF structure with high sensitivity (GF > 1000) but low relatively stretchability (2\%-6\%) [59]. The coordination between high sensitivity and stretchability of FGS that based on fiber assemblies will be a research topic in the future.

\subsection{Linearity}

Linearity is an important parameter for stretchable FGS, because nonlinearity of sensors makes the calibration process complex and difficult for accurate measurement (Fig. 17b). The nonlinearity is caused by the fact that the original homogeneous structure is destroyed and becomes heterogeneous during the strain process [82]. Because the change of fiber assemblies structure, the change of dimension of the fiber, and the change of the connection-disconnection state of graphene sheets are non-synchronous for FGS, which lead the sensitivity index of FGS becoming nonlinear in spite of its stretchable properties. At present, there are few researches on the relationship between the linearity of sensitivity and the fiber assemblies structure. It is necessary to further study how to design fiber assemblies structure to improve the linearity of sensitivity index (Fig. 17c).

\subsection{Hysteresis}

Hysteresis index must be considered for dynamic strain especially for high frequency strain sensing (Fig. 17d). Due to viscoelasticity characteristics of fiber [83], the stress modulus of fiber forming polymer can be divided into dynamic elastic modulus and dynamic loss modulus when applied dynamic force. At the same time, the strain can also be divided into quick elastic strain, slow elastic strain and plastic strain, these are the main causes of hysteresis $[84,85]$. Generally, the hysteresis of the capacitive strain sensor is smaller than that of the piezoresistive strain sensor because the capacitance change is related to the sensing area between the capacitance plate while the resistance change is related to the change of its own resistance of the material $[86,87]$. For the design of the FGS, capacitance type strain sensor can reduce hysteresis because it has little correlation with the properties of the material itself. In addition to the viscoelasticity of fiber, elastic strain 
and plastic strain also existed in the fiber assemblies structure, so the hysteresis of the FGS is inevitable. Therefore, reasonable selection of fiber forming polymer and fiber assemblies structure are the effective ways to reduce the hysteresis of the FGS and to improve the measurement accuracy on high frequency strain.

\subsection{Response and recovery time}

Response time determines how quickly the strain sensors move toward stable response. Due to the viscoelastic properties of the fiber forming polymer, all polymer-based strain sensors have a response delay $[87,88]$. And due to lower effect of viscoelastic properties on capacitive-type strain sensors, it show faster response time than resistive sensors [86]. Recovery time is another important performance parameter of the strain sensor under dynamic stress which caused by the friction force between conductive fillers and polymer matrix. The capacitive-type strain sensor has relatively short recovery time than resistive type one $[2,86]$. For the FGS, the slip of conductive graphene sheets during the process of applying stress are delayed due to the viscoelastic creep characteristics of fiber forming polymers and the friction between the graphene sheets and fiber forming polymer matrix, which result in the response and recovery time. The response and recovery time must be concerned for the detection of high frequency dynamic strain, otherwise, the data collected by the FGS will be distorted.

\subsection{Overshoot behavior}

The stress relaxation of polymers which caused by its viscoelastic properties will lead to the overshoot behavior of sensors $[89,90]$. When the strain is suddenly reserved at the end of the stretching cycle, polymers intend to release their stress instantly by molecular or molecular segment motions. These internal structural changes may not affect the permittivity of the dielectric layer considerably which lead to a small overshoot behavior to capacitive-type strain sensors. On the other hand, this deformation may change the resistance of resistive-type sensor, causing a relatively large overshoot behavior. For the FGS the structure changed by the relaxation of fiber forming polymer lead to the connection-disconnection state of graphene sheets, so the overshoot behavior should not be neglected. There are few studies on this area till now.

\subsection{Durability}

Durability represents the endurance of sensors on the long-term stressing/releasing cycles. To have stable sensing performance during long-term stressing/releasing cycles is important for wearable sensors, because it has to accommodate large, complex, and dynamic strains while wearing. The degradation of the sensing performance of sensors were caused by the fatigue and plastic deformation of polymer substrates or caused by the interfacial performance degradation between conductive nanofiller and polymer substrates under long-term stressing/releasing cycles, such as the decrease of sensitivity and the increase of hysteresis time etc. [91]. For the FGS, to improve the fatigue resistance of fiber forming polymer and the interfacial performance between fiber and graphene will improve the durability. Although the durability of reported FGS was more than 1000 times, its sensing indexes have deteriorated due to the degradation of the interfacial properties between graphene and the fiber forming polymer substrate $[62,81]$. Therefore, improving the durability of the FGS to retain a stable sensing performance is worthy to be further studied. $[92,93]$

In general, FGS usually have properties of good stretchability, high sensitivity, and low linearity. At the same time, hysteresis, response and recovery time, and overshoot behavior are inevitable due to the viscoelasticity of the fiber forming polymers that used as FGS substrate, so its influence on the sensing accuracy can only be reduced or corrected as much as possible. In addition, its durability also need to be further improved. Table 1 listed the main sensing performance indexes of the FGS that had been reported previously.

\section{Application}

The FGS are mainly classified into two categories. One is with high stretchability and relatively low sensitivity, which can be used to detect large strain such as gesture recognition, body movements, position measurement, step measurement. The other is with high sensitivity and relatively low stretchability, which can be used to detect the small strain such as voice and expression recognition, heartbeat and pulse measurement, physical indicators monitoring. As shown in Fig. 18, Park et al. fabricated a FGS based on yarn structure by LBL dip-coating graphene dispersion on nylon filaments as follow: firstly coating a layer of polyvinyl alcohol (PVA) on the surface of the nylon filaments, and then dip-coating a layer of graphene nanosheets dispersion, after multiple layers of dip-coating, a PDMS elastic film was used to cover on the outer layer. The prepared FGS with high stretchable (strain $>150 \%$ ) to monitor movements in various parts of the body [1]. As shown in Fig. 19, Cheng et al. used polyester/spandex core-spun yarns as substrates, firstly coated a layer of GO by dip-coating then reduced $\mathrm{GO}$ to $\mathrm{rGO}$. The prepared FGS has advantages of relatively 
Table 1 Fabrication and main sensing performance indexes of reported FGS

\begin{tabular}{|c|c|c|c|c|c|c|}
\hline Substrate & Combination & Stretchability (\%) & Sensitivity GF & Durability (times) & Application & References \\
\hline $\begin{array}{l}\text { PA6 } \\
\text { Filament }\end{array}$ & Dip-coating & $<150$ & 2 & 120 & Human motion monitoring & [1] \\
\hline Sheath-core fiber & Dry-coating & 15 & 14.5 & 3000 & Finger recognition & {$[22]$} \\
\hline $\begin{array}{l}\text { PET/PU } \\
\text { Core-spun yarn }\end{array}$ & Dip-coating & $\begin{array}{l}<1 \\
<50\end{array}$ & $\begin{array}{l}10 \\
3.7\end{array}$ & 1000 & $\begin{array}{l}\text { Heart beat Detection } \\
\text { Voice recognition }\end{array}$ & [94] \\
\hline $\begin{array}{l}\text { TPU } \\
\text { Fibrous mats }\end{array}$ & Dip-coating & $\begin{array}{l}10 \\
100\end{array}$ & $\begin{array}{l}11 \\
79\end{array}$ & 250 & Human motion & {$[95]$} \\
\hline GWF & CVD & $\begin{array}{l}<0.2 \\
2-6\end{array}$ & $\begin{array}{l}35 \\
1000\end{array}$ & -150 & Expression/Voice recognition & [59] \\
\hline GWF & CVD & $\begin{array}{l}<2 \\
2-6\end{array}$ & $\begin{array}{l}500 \\
1000\end{array}$ & 20 & Pulse measurement & {$[71]$} \\
\hline PU fabric & Dip-coating & $<40$ & 67.3 & 70 & $\begin{array}{l}\text { Movement monitoring } \\
\text { Gesture recognition }\end{array}$ & [57] \\
\hline $\begin{array}{l}\text { PA6/PU } \\
\text { fabric }\end{array}$ & Dip-coating & $\begin{array}{l}<10 \\
10-20\end{array}$ & $\begin{array}{l}18.5 \\
12.1\end{array}$ & 120 & Finger recognition & {$[75]$} \\
\hline Cotton bandages & filtration & $\begin{array}{l}<40 \\
<57\end{array}$ & $\begin{array}{l}416 \\
3367\end{array}$ & 250 & Voice recognition pulse measurement & {$[62]$} \\
\hline
\end{tabular}
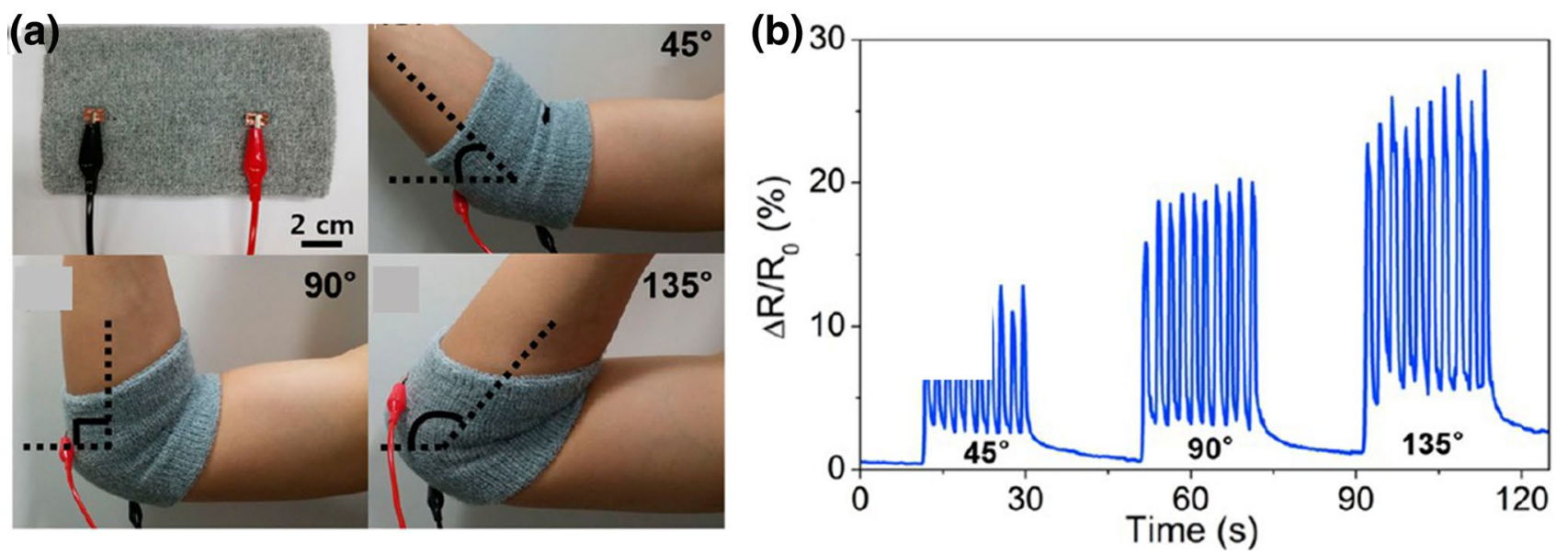

Fig. 18 a Photographs of the FGS based on nylon yarn that sewed in an elbow wrap to monitor the bending motion of arm at different angles of $45^{\circ}, 90^{\circ}$, and $135^{\circ}$. b Relative resistance change of the FGS according to the bending motions [1]

highly sensitivity at small strain (GF 10 at strain 1\%), short response time (<100 ms), good durability ( $>1000$ cycles), so it can be used to detect human movement of walking, squatting and sound recognition [94]. As shown in Fig. 20, Wang et al. reported a FGS based on GWF structure, firstly made a GWF structure by CVD growing method and then transferred GWF to PDMS elastic structure and encapsulated into a FGS. The prepared FGS with ultra-sensitivity under small strain (GF 35 at strain $<0.2 \%$ ) can be used to detect very small strain such as facial expressions and eye blink [59]. As shown in Fig. 21, Yang et al. used the same method to prepare FGS based on GWF structure, which has ultra-high sensitivity under small strain (GF 20 at strain $<0.1 \%$ ). It is similar to the tactile sensation of artificial skin and can accurately perceive the difference of pulse in three adjacent positions to diagnose human health utilizing the theory of traditional Chinese medicine [82]. As shown in 
(a)

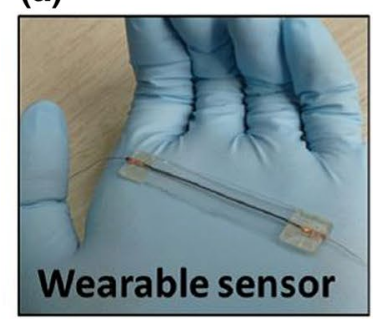

(e)

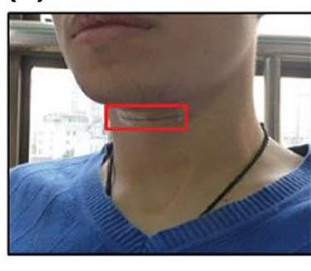

(b)

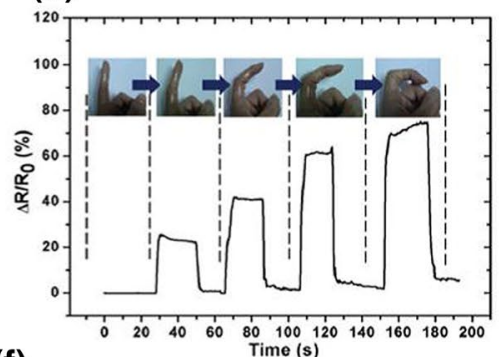

(f)

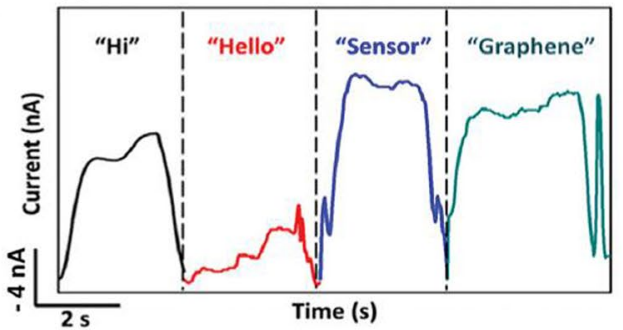

(c)

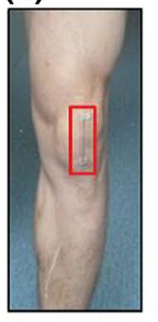

(g)

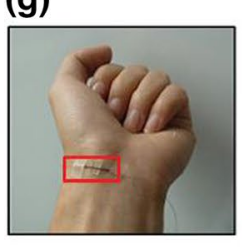

(d)

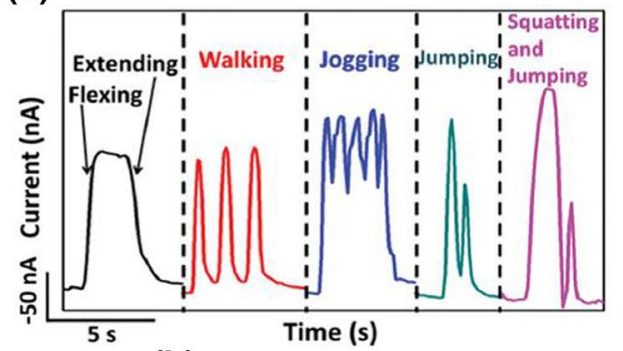

(h)

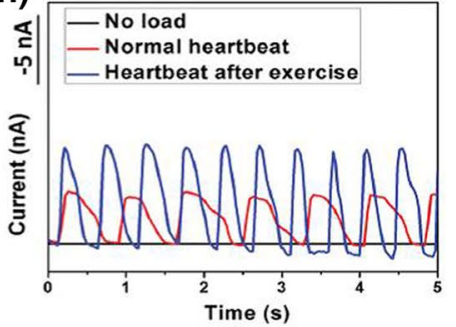

Fig. 19 The Applications of FGS for human movement monitoring. a Photograph of the FGS. b Responsive signal of FGS in monitoring finger bending to corresponding positions. c FGS attached to the knee. $\mathbf{d}$ Responsive curves of FGS on the knee under motions of flexing/extending, walking, jogging, jumping, and squatting-jump- ing. e FGS attached to the throat. $\mathbf{f}$ Responsive curves when wearer spoke "Hi", "Hello", "Sensor", and "Graphene." g FGS attached to the wrist. $\mathbf{h}$ Responsive curves of FGS on the wrist before wearing (no load), and under normal/exercise conditions [94]
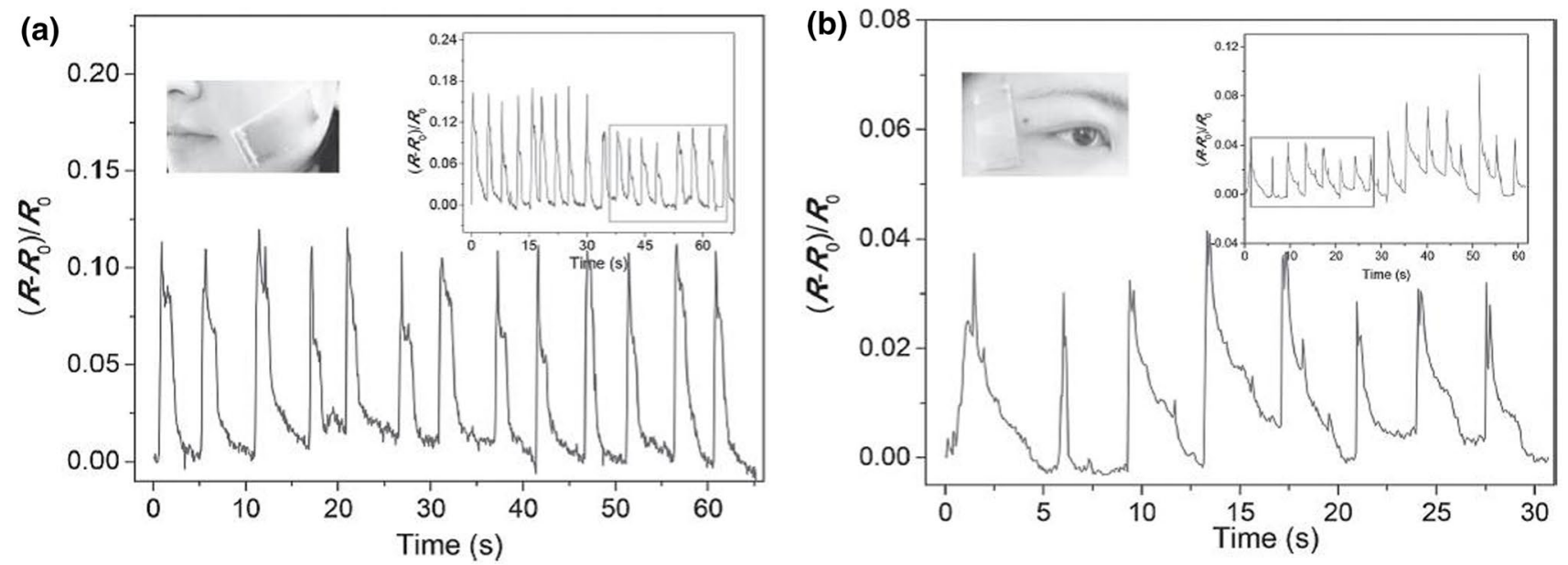

Fig. 20 Sensing performance of FGS in muscle motions of expression changes and blinks in (a) and (b) respectively [59]

Fig. 22, Lou et al. fabricated a FGS that based on fabric structure, firstly combined the $\mathrm{GO}$ with polyester fabric by dip-coating method and then reduced $\mathrm{GO}$ to $\mathrm{rGO}$. The prepared FGS with good piezoresistive sensitivity ( $\mathrm{K}$ $0.012 \mathrm{KPa}^{-1}$ at pressure $800 \mathrm{KPa}$ ) and very fast response time ( $50 \mathrm{~ms}$ ) can be used to detect the plantar pressure distribution on ground [97]. Although the application of FGS in the monitoring of human exercise, health and physiological indicators is still in-depth research, it is sufficient to confirm the prospect of future development through the above application examples. 
Fig. 21 Multiposition pulse reading by FGS. a Location of the inch opening. $\mathbf{b}$ Pulse signals located at the Cun (distal), Guan (middle), and Chi (proximal) positions [82]
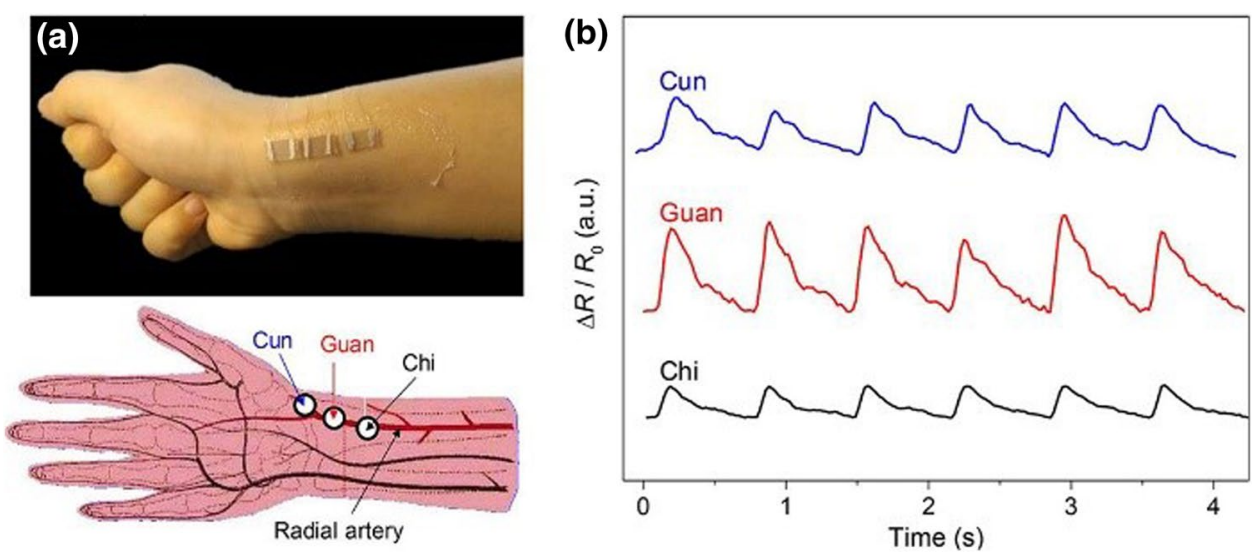

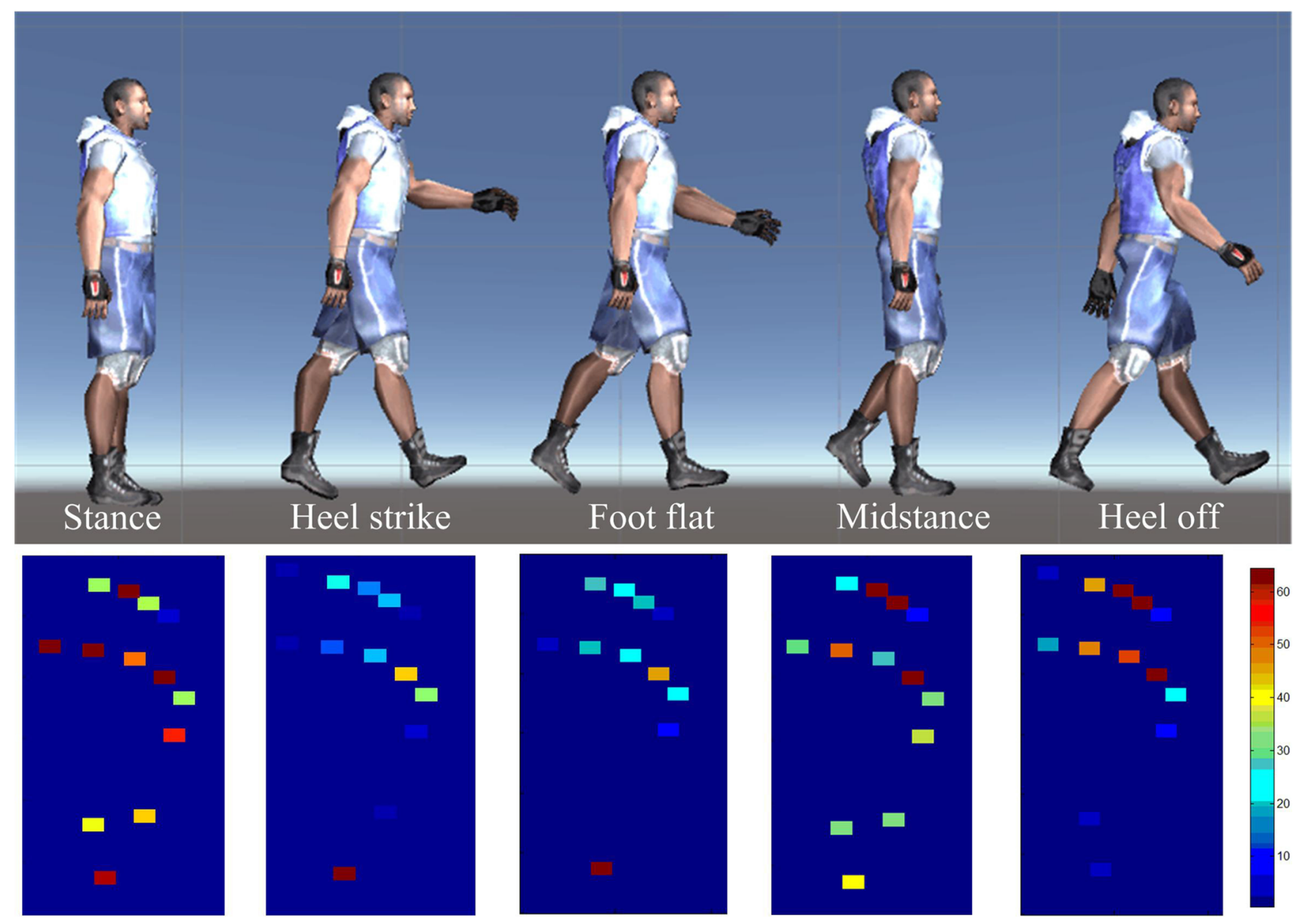

Fig. 22 Plantar pressure distribution of right foot in the major phase of one full gait cycle [97]

Integration of graphene with fiber assemblies will inevitably promote the rapid development and widespread applications of wearable electronics. Each of the sensors listed above has its own advantages, however, what we still need to improve is to get the high stretchability and high sensitivity in the meantime. It is very important for the innovation of the application of FGS. 


\section{Conclusion}

Herein, we have conducted a comprehensive investigation and detailed discussion on current wearable graphene strain sensor based on fiber assemblies (FGS), including graphene preparation, preparation of FGS as well as its sensing mechanism, main sensing performance and application. Although fiber assemblies as daily used textilerelated materials exhibits outstanding ability of structural retention and fatigue resistance during wearing and washing, which can provide a large-area affiliated carrier and widespread service platform for the design and application of functional electronics textiles, and the graphene with good electrical and mechanical properties can serve as an effective sensing sensitive material, there are still three challenges to be further studied. (i) How to reduce the effects of intrinsic viscoelastic characteristics of fibers on sensing performance, especially under dynamic stress. (ii) How to improve the interaction between graphene and fiber assemblies in order to improve sensing performance. (iii) How to design fiber assemblies structure to monitor various strains. Although these challenges mentioned in this work have seriously hindered the development process of FGS, the hotspots on research and widespread practical application of them are an irresistible trend in the near future. We believe that with further research and technological advances, these problems will be solved and the application of FGS will eventually take into practice.

Acknowledgements The work was guided by Intelligent Wearable Technology Research Center of Qingdao University and The Key Laboratory of Advanced Textile Materials and Manufacturing Technology of Ministry of Education, Zhejiang Sci-Tech.

Author contributions This work presented here was performed in collaboration with all the authors. All authors read and approved the final manuscript. Yixin Liu had the idea for the article, Zhaofeng Ouyang and Fei Wang performed the literature search and data analysis, and Ting Wang drafted the work, Yixin Liu critically revised the work.

\section{Compliance with ethical standards}

Conflict of interest The authors declare that they have no conflict of interest.

\section{References}

1. Park JJ, Hyun WJ, Mun SC, Park YT, Park OO (2015) Highly stretchable and wearable graphene strain sensors with controllable sensitivity for Human motion monitoring. ACS Appl Mater Int 7:6317-6324. https://doi.org/10.1021/acsami.5b00695

2. Lin MF, Xiong J, Wang J, Parida K, Lee PS (2018) Core-shell nanofiber mats for tactile pressure sensor and nanogenerator applications. Nano Energy 44:248-255. https://doi. org/10.1016/j.nanoen.2017.12.004
3. Li L, Lou Z, Chen D, Jiang K, Han W, Shen G (2017) Recent advances in fexible/stretchable supercapacitors for wearable electronics. Small. https://doi.org/10.1002/smll.201702829

4. Heo JS, Eom J, Kim Y-H, Park SK (2018) Recent progress of textilebased wearable electronics: a comprehensive review of materials, devices, and applications. Small 14:1703034. https://doi. org/10.1002/smll.201703034

5. Wan Y, Wang Y, Guo CF (2017) Recent progresses on flexible tactile sensors. Mater Today Phys 1:61-73. https://doi.org/10.1016/j. mtphys.2017.06.002

6. Cao Z, Wang R, He T, Xu F, Sun J (2018) Interface-controlled conductive fibers for wearable strain sensors and stretchable conducting wires. ACS Appl Mater Interfaces 10:14087. https:// doi.org/10.1021/acsami.7b19699

7. Souri H, Bhattacharyya D (2018) Wearable strain sensors based on electrically conductive natural fiber yarns. Mater Des 154:217-227. https://doi.org/10.1016/j.matdes.2018.05.040

8. He X, Zi Y, Guo H, Zheng H, Xi Y, Wu C, Wang J, Zhang W, Lu C, Wang ZL (2017) A highly stretchable fiber-based triboelectric nanogenerator for self-powered wearable electronics. Adv Funct Mater 27:1604378. https://doi.org/10.1002/adfm.20160 4378

9. Pu X, Li L, Liu M, Jiang C, Du C, Zhao Z (2016) Wearable selfcharging power textile based on flexible yarn supercapacitors and fabric nanogenerators. Adv Mater 28:98-105. https://doi. org/10.1002/adma.201504403

10. Li X, Hua T, Xu B (2017) Electromechanical properties of a yarn strain sensor with graphene-sheath/polyurethane-core. Carbon 118:686-698. https://doi.org/10.1016/j.carbon.2017.04.002

11. Liu Y, Liu L, Li Z et al (2020) 3D network structure and sensing performance of woven fabric as promising flexible strain sensor. SN Appl Sci 2:70. https://doi.org/10.1007/s42452-019-1857-6

12. Seyedin S, Moradi S, Singh C, Razal JM (2018) Continuous production of stretchable conductive multifilaments in kilometer scale enables facile knitting of wearable strain sensing textiles. Appl Mater Today 11:255-263. https://doi.org/10.1016/j. apmt.2018.02.012

13. Liu H, Li Q, Bu Y, Zhang N, Wang C, Pan C et al (2019) Stretchable conductive nonwoven fabrics with self-cleaning capability for tunable wearable strain sensor. Nano Energy. https://doi. org/10.1016/j.nanoen.2019.104143

14. Gong XX, Fei GT, Fu WB, Fang M, Gao XD, Zhong BN, Zhang LD (2017) Flexible strain sensor with high performance based on PANI/PDMS films. Org Electron 47:51-56. https://doi. org/10.1016/j.orgel.2017.05.001

15. Hao D, Xu B, Cai Z (2018) Polypyrrole coated knitted fabric for robust wearable sensor and heater. J Mater Sci 29:9218-9226. https://doi.org/10.1007/s10854-018-8950-2

16. Seyedin S, Razal JM, Innis PC, Jeiranikhameneh A, Beirne S, Wallace GG (2015) Knitted strain sensor textiles of highly conductive all-polymeric fibers. Acs Appl Mater Interfaces 7:21150. https:// doi.org/10.1021/acsami.5b04892

17. Duan L, Spoerk M, Wieme T, Cornillie P, Xia H, Zhang J et al (2019) Designing formulation variables of extrusion-based manufacturing of carbon black conductive polymer composites for piezoresistive sensing. Compos Sci Technol 171:78-85. https:// doi.org/10.1016/j.compscitech.2018.12.009

18. Li W, Xu F, Liu W, Gao Y, Zhang K, Zhang X, Qiu Y (2018) Flexible strain sensor based on aerogel-spun carbon nanotube yarn with a core-sheath structure. Composites A 108:107-113. https://doi. org/10.1016/j.compositesa.2018.02.024

19. Niu D, Jiang W, Ye G, Wang K, Yin L, Shi Y, Chen B, Lou F, Liu H (2018) Graphene-elastomer nanocomposites based flexible piezoresistive sensors for strain and pressure detection. Mater Res Bull 102:92-99. https://doi.org/10.1016/j.materresbull.2018.02.005 
20. Ni GX, McLeod AS, Sun Z et al (2018) Fundamental limits to graphene plasmonics. Nature 557:530-533. https://doi. org/10.1038/s41586-018-0136-9

21. Lee C, Wei X, Kysar JW, Hone J (2008) Measurement of the elastic properties and intrinsic strength of monolayer graphene. Science 321:385-388. https://doi.org/10.1126/science.1157996

22. Zhang M, Wang C, Wang Q, Jian M, Zhang Y (2016) Sheath-core graphite/silk fiber made by dry-meyer-rod-coating for wearable strain sensors. ACS Appl Mater Interfaces 8:20894-20899. https ://doi.org/10.1021/acsami.6b06984

23. Liu X, Tang C, Du X, Xiong S, Xi S, Liu Y, Shen X, Zheng Q, Wang Z, Wu Y, Horner A, Kim JK (2017) A highly sensitive graphene woven fabric strain sensor for wearable wireless musical instruments. Mater Horiz 4(3):477-486. https://doi.org/10.1039/ c7mh00104e

24. Boukhvalov DW, Katsnelson MI, Lichtenstein Al (2008) Hydrogen on graphene: electronic structure, total energy, structural distortions and magnetism from first-principles calculations. Phys Rev B 77(3):35427. https://doi.org/10.1103/physrevb.77.035427

25. Liu S, Chevali VS, Xu Z, Hui D, Wang H (2018) A review of extending performance of epoxy resins using carbon nanomaterials. Composites B 136:197-214. https://doi.org/10.1016/j.compo sitesb.2017.08.020

26. Kundu S, Sriramdas R, Rafsanjani AK, Bid A, Pratap R, Ravishankar N (2017) Crumpled sheets of reduced graphene oxide as a highly sensitive, robust and versatile strain/pressure sensor. Nanoscale 9(27):9581-9588. https://doi.org/10.1039/c7nr0 $2415 \mathrm{k}$

27. Nie M, Xia Y, Yang H (2018) A flexible and highly sensitive graphene-based strain sensor for structural health monitoring. Clust Comput 01:1-8. https://doi.org/10.1007/s1058 6-018-1727-9

28. Nie M, Yang HS, Xia YH (2018b) Graphene based strain sensor with LCP substrate IOP Publishing Materials Science and Engineering https://iopscience.iop.org/article/10.1088/1757899X/307/1/012051/meta. Accessed 2018

29. Tiefenauer RF, Dalgaty $T$, Keplinger T, Tian T, Shih CJ, Vörös $J$ (2018) Monolayer graphene coupled to a flexible plasmonic nanograting for ultrasensitive strain monitoring. Small 14:e1801187. https://doi.org/10.1002/smll.201801187

30. Tung TT, Nine MJ, Krebsz M, Pasinszki T, Coghlan CJ, Tran DNH, Losic D (2017) Recent advances in sensing applications of graphene assemblies and their composites. Adv Funct Mater 27:1702891. https://doi.org/10.1002/adfm.201702891

31. Cui G, Bi Z, Zhang R, Liu J, Yu X, Li Z (2019) A comprehensive review on graphene-based anti-corrosive coatings. Chem Eng J 373(2019):104-121. https://doi.org/10.1016/j.cej.2019.05.034

32. Bo Z, Shuai X, Mao S, Yang H, Qian J, Chen J, Yan J, Cen K (2014) Green preparation of reduced graphene oxide for sensing and energy storage applications. Sci Rep 4:4684. https://doi. org/10.1038/srep04684

33. Muthoosamy K, Manickam S (2017) State of the art and recent advances in the ultrasound-assisted synthesis, exfoliation and functionalization of graphene derivatives. Ultrason Sonochem 39:478. https://doi.org/10.1016/j.ultsonch.2017.05.019

34. Nakamura D, Nakano H (2018) Liquid-Phase exfoliation of germanane based on hansen solubility parameters. Chem Mater 30:5333-5338. https://doi.org/10.1021/acs.chemmater.8b021 53

35. Xia ZY, Pezzini S, Treossi E, Giambastiani G, Corticelli F, Morandi V, Zanelli A, Bellani V, Palermo V (2013) The exfoliation of graphene in liquids by electrochemical, chemical, and sonication-assisted techniques: a nanoscale study. Adv Funct Mater 23:4684-4693. https://doi.org/10.1002/adfm.201203686

36. Bae S, Kim H, Lee $Y, X u$ X, Park J-S, Zheng $Y$ et al (2010) Rollto-roll production of 30 -inch graphene films for transparent electrodes. Nat Nanotechnol 5:574. https://doi.org/10.1038/ nnano.2010.132

37. Jang H, Park YJ, Chen X, Das T, Kim MS, Ahn JH (2016) Graphenebased flexible and stretchable electronics. Adv Mater 28:41844202. https://doi.org/10.1002/adma.201504245

38. Lavin-Lopez MP, Valverde JL, Sanchez-Silva L, Romero A (2016) Solvent-based exfoliation via sonication of graphitic materials for graphene manufacture. Ind Eng Chem Res 55:845-855. https ://doi.org/10.1021/acs.iecr.5b03502

39. Hernandez Y, Lotya M, Rickard D, Bergin SD, Coleman JN (2010) Measurement of multicomponent solubility parameters for graphene facilitates solvent discovery. Langmuir 26:3208-3213. https://doi.org/10.1021/la903188a

40. Khan U, O'Neill A, Lotya M, De S, Coleman JN (2010) High-concentration solvent exfoliation of graphene. Small 6:864-871. https://doi.org/10.1002/smll.200902066

41. Liu N, Luo F, Wu H, Liu Y, Zhang C, Chen J (2008) One-Step ionicliquid-assisted electrochemical synthesis of ionic-liquid-functionalized graphene sheets directly from graphite. Adv Funct Mater 18:1518-1525. https://doi.org/10.1002/adfm.200700797

42. Xu Z, Gao C (2015) Graphene fiber: a new trend in carbon fibers. Mater Today 18:480-492. https://doi.org/10.1016/j.matto d.2015.06.009

43. Meng F, Lu W, Li Q, Byun JH, Oh Y, Chou TW (2015) Graphenebased fibers: a review. Adv Mater 27:5113-5131. https://doi. org/10.1002/adma.201501126

44. Jalili R, Aboutalebi SH, Esrafilzadeh D, Shepherd RL, Chen J, Aminorroaya-Yamini S, Konstantinov K, Minett Al, Razal JM, Wallace GG (2013) Scalable one-step wet-spinning of graphene fibers and yarns from liquid crystalline dispersions of graphene oxide: towards multifunctional textiles. Adv Funct Mater 23:53455354. https://doi.org/10.1002/adfm.201300765

45. Montes I, Lai C, Sanabria D (2003) Like dissolves like: a guided inquiry experiment for organic chemistry. J Chem Educ 80:447. https://doi.org/10.1021/ed080p447

46. Cong HP, Ren XC, Wang P, Yu SH (2012) Wet-spinning assembly of continuous, neat, and macroscopic graphene fibers. Sci Rep 2:613. https://doi.org/10.1038/srep00613

47. Sun J, Li Y, Peng Q, Hou S, Zou D, Shang Y, Li Y, Li P (2013) Macroscopic, flexible, high-performance graphene ribbons. ACS Nano 7:10225-10232. https://doi.org/10.1021/nn404533r

48. Kou L, Huang T, Zheng B, Han Y, Zhao X (2014) Coaxial wet-spun yarn supercapacitors for high-energy density and safe wearable electronics. Nat Commun 5:3754. https://doi.org/10.1038/ ncomms4754

49. Zhao Y, Jiang C, Hu C, Dong Z, Xue J, Meng Y (2013) Large-scale spinning assembly of neat, morphology-defined, graphenebased hollow fibers. ACS Nano 7:2406-2412. https://doi. org/10.1021/nn305674a

50. Aboutalebi SH, Jalili R, Esrafilzadeh D, Salari M, Gholamvand Z, Aminorroaya Yamini S, Konstantinov K, Shepherd RL, Chen J, Moulton SE, Charles Innis P, Minett Al, Razal JM, Wallace GG (2014) High-performance multifunctional graphene yarns: toward wearable all-carbon energy storage textiles. ACS Nano 8:2456-2466. https://doi.org/10.1021/nn406026z

51. Xu Z, Sun H, Zhao X, Gao C (2013) Ultrastrong fibers assembled from giant graphene oxide sheets. Adv Mater 25:188-193. https ://doi.org/10.1002/adma.201203448

52. Dong Z, Jiang C, Cheng H, Zhao Y, Shi G, Jiang L, Qu L (2012) Facile fabrication of light, flexible and multifunctional graphene fibers. Adv Mater 24:1856-1861. https://doi.org/10.1002/ adma.201200170

53. Li J, Li J, Li L, Yu M, Ma H, Zhang B (2014) Flexible graphene fibers prepared by chemical reduction-induced self-assembly. J Mater Chem A 2:6359-6362. https://doi.org/10.1039/c4ta00431k 
54. Li X, Zhao T, Wang K, Yang Y, Wei J, Kang F, Wu D (2011) Directly drawing self-assembled, porous, and monolithic graphene fiber from chemical vapor deposition grown graphene film and its electrochemical properties. Langmuir 27:12164-12171. https ://doi.org/10.1021/la202380g

55. Li Q, Ullah Z, Li W, Guo Y, Xu J, Wang R, Zeng Q (2016) Widerange strain sensors based on highly transparent and supremely stretchable graphene/Ag-nanowires hybrid structures. Small 12:5058-5065. https://doi.org/10.1002/smll.201600487

56. Shi J, Li X, Cheng H, Liu Z, Zhao L, Yang T, Dai Z, Cheng Z, Shi E, Yang L, Zhang Z, Cao A, Zhu H, Fang Y (2016) Graphene reinforced carbon nanotube networks for wearable strain sensors. Adv Funct Mater 26:2078-2084. https://doi.org/10.1002/ adfm.201504804

57. Huang Y, Gao L, Zhao Y, Guo X, Liu C, Liu P (2017) Highly flexible fabric strain sensor based on graphene nanoplatelet-polyaniline nanocomposites for human gesture recognition. J Appl Polym Sci 134(39):45340. https://doi.org/10.1002/app.45340

58. Kou L, Gao C (2013) Bioinspired design and macroscopic assembly of poly(vinyl alcohol)-coated graphene into kilometers-long fibers. Nanoscale 5:4370-4378. https://doi.org/10.1039/C3NR00455D

59. Wang Y, Wang L, Yang T, Li X, Zang X, Zhu M, Wang K, Wu D, Zhu $H(2014)$ Wearable and highly sensitive graphene strain sensors for human motion monitoring. Adv Funct Mater 24(29):46664670. https://doi.org/10.1002/adfm.201400379

60. Molina J (2016) Graphene-based fabrics and their applications: a review. RSC Adv 6(72):68261-68291. https://doi.org/10.1039/ c6ra12365a

61. Du D, Li P, Ouyang J (2016) Graphene coated nonwoven fabrics as wearable sensors. J Mater Chem C 4(15):3224-3230. https:// doi.org/10.1039/c6tc00350h

62. Yin B, Wen Y, Hong T, Xie Z, Yuan G, Ji Q, Jia H (2017) Highly stretchable, ultrasensitive, and wearable strain sensors based on facilely prepared reduced graphene oxide woven fabrics in an ethanol flame. Acs Appl Mater Interfaces 9:32054. https://doi. org/10.1021/acsami.7b09652

63. Secor EB, Prabhumirashi PL, Puntambekar K, Geier ML, Hersam MC (2013) Inkjet printing of high conductivity, flexible graphene patterns. J Phys Chem Lett 4:1347-1351. https://doi. org/10.1021/jz400644c

64. Karim N, Afroj S, Malandraki A, Butterworth S, Beach C, Rigout M, Novoselov KS, Casson AJ, Yeates SG (2017) All inkjet-printed graphene-based conductive patterns for wearable e-textile applications. J Mater Chem C 5(44):11640-11648. https://doi. org/10.1039/c7tc03669h

65. Amjadi M, Kyung K-U, Park I, Sitti M (2016) Stretchable, skinmountable, and wearable strain sensors and their potential applications: a review. Adv Funct Mater 26:1678-1698. https:// doi.org/10.1002/adfm.201504755

66. Ma H, Wu W, Cao J, Yue B, Zhang H (2016) Network structure and electromechanical properties of viscose-graphene conductive yarn assembles. Carbon 114:731-739. https://doi.org/10.1016/j. carbon.2016.12.063

67. Zhao J, Zhang GY, Shi DX (2013) Review of graphene-based strain sensors. Chin Phys B 22(5):057701

68. Adams JD, Schwalb CH, Winhold M, Huth M, Đukić M, Fantner GE (2013) Analysis of local deformation effects in resistive strain sensing of a submicron-thickness AFM cantilever. Smart Sens Actuators MEMS VI. https://doi.org/10.1117/12.2018034

69. Lu Y, Biswas MC, Guo Z, Jeon J-W, Wujcik EK (2018) Recent developments in bio-monitoring via advanced polymer nanocomposite-based wearable strain sensors. Biosens Bioelectron. https ://doi.org/10.1016/j.bios.2018.08.037

70. Desai AV, Haque MA (2005) Mechanics of the interface for carbon nanotube-polymer composites. Thin Wall Struct 43:17871803. https://doi.org/10.1016/j.tws.2005.07.003
71. Yang T, Wang W, Zhang H, Li X, Shi J, He Y, Zhu H (2015) Tactile sensing system based on arrays of graphene woven microfabrics: electromechanical behavior and electronic skin application. ACS Nano 9(11):10867-10875. https://doi.org/10.1021/acsna no. 5 b03851

72. Chun S, Jung H, Choi Y, Bae G, Kil JP, Park W (2015) A tactile sensor using a graphene film formed by the reduced graphene oxide flakes and its detection of surface morphology. Carbon 94:982-987. https://doi.org/10.1016/j.carbon.2015.07.088

73. Tang Y, Zhao Z, Hu H, Liu Y, Wang X, Zhou S, Qiu J (2015) Highly stretchable and ultrasensitive strain sensor based on reduced graphene oxide microtubes-elastomer composite. ACS Appl Mater Inter 7:27432-27439. https://doi.org/10.1021/acsam i.5b09314

74. Kang D, Pikhitsa PV, Choi YW, Lee C, Shin SS, Piao L (2014) Ultrasensitive mechanical crack-based sensor inspired by the spider sensory system. Nature 516:222. https://doi.org/10.1038/natur e14002

75. Cai G, Yang M, Xu Z, Liu J, Tang B, Wang X (2017) Flexible and wearable strain sensing fabrics. Chem Eng J 325:396-403. https ://doi.org/10.1016/j.cej.2017.05.091

76. Sun Q, Seung W, Kim BJ, Seo S, Kim SW, Cho JH (2015) Active matrix electronic skin strain sensor based on piezopotentialpowered graphene transistors. Adv Mater 27:3411-3417. https ://doi.org/10.1002/adma.201500582

77. Atalay O, Kennon WR, Husain MD (2013) Textile-based weft knitted strain sensors: effect of fabric parameters on sensor properties. Sensors 13:11114-11127. https://doi.org/10.3390/s1308 11114

78. Lu Y, Tian M, Sun X, Pan N, Chen F, Zhu S et al (2019) Highly sensitive wearable $3 \mathrm{D}$ piezoresistive pressure sensors based on graphene coated isotropic non-woven substrate. Composites A 117:202-210. https://doi.org/10.1016/j.composites a.2018.11.023

79. Büscher GH, Kõiva R, Schürmann C, Haschke R, Ritter HJ (2015) Flexible and stretchable fabric-based tactile sensor. Robot Auton Syst 63:244-252. https://doi.org/10.1016/j.robot.2014.09.007

80. Lee H, Glasper MJ, Li X, Nychka JA, Batcheller J, Chung HJ, Chen $Y(2018)$ Preparation of fabric strain sensor based on graphene for human motion monitoring. J Mater Sci 53:9026-9033. https ://doi.org/10.1007/s10853-018-2194-7

81. Xie J, Long $\mathrm{H}$, Miao $\mathrm{M}$ (2016) High sensitivity knitted fabric strain sensors. Smart Mater Struct 25:105008. https://doi. org/10.1088/0964-1726/25/10/105008

82. Yang T, Jiang $X$, Zhong Y, Zhao X, Lin S, Li J et al (2017) A wearable and highly sensitive graphene strain sensor for precise home-based pulse wave monitoring. ACS Sens 2:967-974. https ://doi.org/10.1021/acssensors.7b00230

83. Muliana AH (2010) A micromechanical formulation for piezoelectric fiber composites with nonlinear and viscoelastic constituents. Acta Mater 58:3332-3344. https://doi.org/10.1016/j. actamat.2010.02.007

84. Banks HT (2008) A brief review of some approaches to hysteresis in viscoelastic polymers. Nonlinear Anal-Theor 69(3):807-815. https://doi.org/10.1016/j.na.2008.02.103

85. Chu CY, Yu WD (2009) Textile physicals. Donghua University Press, China

86. Cai L, Song L, Luan P, Zhang Q, Zhang N, Gao Q, Zhao D, Zhang $X$, Tu M, Yang F (2013) Super-stretchable, transparent carbon nanotube-based capacitive strain sensors for human motion detection. Sci Rep 3:3048. https://doi.org/10.1038/srep03048

87. Gong S, Lai DTH, Su B, Si KJ, Ma Z, Yap LW, Guo P, Cheng W (2015) Highly stretchy black gold e-skin nanopatches as highly sensitive wearable biomedical sensors. Adv Electron Mater 1:1400063. https://doi.org/10.1002/aelm.201400063 
88. Srivastava I, Yu ZZ, Koratkar NA (2012) Viscoelastic properties of graphene-polymer composites. Adv Sci Eng Med 4:10-14. https ://doi.org/10.1166/asem.2012.1127

89. Zu M, Li Q, Zhu Y, Zhu Y, Wang G, Byun J-H, Chou T-W (2013) Stress relaxation in carbon nanotube-based fibers for load-bearing applications. Carbon 52:347-355. https://doi.org/10.1016/j. carbon.2012.09.036

90. Yan T, Wang Z, Wang Y-Q, Pan Z-J (2018) Carbon/graphene composite nanofiber yarns for highly sensitive strain sensors. Mater Des 143:214-223. https://doi.org/10.1016/j.matdes.2018.02.006

91. Amjadi M, Pichitpajongkit A, Lee S, Ryu S, Park I (2014) Highly stretchable and sensitive strain sensor based on silver nanowire-elastomer nanocomposite. ACS Nano 8:5154-5163. https:// doi.org/10.1021/nn501204t

92. Lin S, Zhao X, Jiang X, Wu A, Ding H, Zhong $Y$ et al (2019) Highly stretchable, adaptable, and durable strain sensing based on a bioinspired dynamically cross-linked graphene/polymer composite. Small. https://doi.org/10.1002/smll.201900848

93. Shi G, Zhao Z, Pai JH, Lee I, Zhang L, Stevenson C, Ishara K, Zhang R, Zhu H, Ma J (2016) Highly sensitive, wearable, durable strain sensors and stretchable conductors using graphene/silicon rubber composites. Adv Funct Mater 26:7614-7625. https:// doi.org/10.1002/adfm.201602619
94. Cheng Y, Wang R, Sun J, Gao L (2015) A stretchable and highly sensitive graphene-based fiber for sensing tensile strain, bending, and torsion. Adv Mater 27:7365-7371. https://doi. org/10.1002/adma.201503558

95. Wang Y, Hao J, Huang Z, Zheng G, Dai K, Liu C, Shen C (2017) Flexible electrically resistive-type strain sensors based on reduced graphene oxide-decorated electrospun polymer fibrous mats for human motion monitoring. Carbon 126:360371. https://doi.org/10.1016/j.carbon.2017.10.034

96. Jeong YR, Park H, Jin SW, Hong SY, Lee S-S, Ha JS (2015) Highly stretchable and sensitive strain sensors using fragmentized graphene foam. Adv Funct Mater 25:4228-4236. https://doi. org/10.1002/adfm.201501000

97. Lou C, Wang S, Liang T, Pang C, Huang L, Run M, Liu X (2017) A graphene-based flexible pressure sensor with applications to plantar pressure measurement and gait analysis. Materials 10(9):1068. https://doi.org/10.3390/ma10091068

Publisher's Note Springer Nature remains neutral with regard to jurisdictional claims in published maps and institutional affiliations. 